\title{
Spatio-Temporal Decomposition and Coordination for Distributed Load Restoration in AC/DC Hybrid System
}

Zhao, Jin; Wang, Hongtao ; Wu, Qiuwei; Hatziargyriou, Nikos D.; Zhang, Wen

Published in:

IEEE Transactions on Smart Grid

Link to article, DOI:

10.1109/TSG.2020.3025694

Publication date:

2021

Document Version

Peer reviewed version

Link back to DTU Orbit

Citation (APA):

Zhao, J., Wang, H., Wu, Q., Hatziargyriou, N. D., \& Zhang, W. (2021). Spatio-Temporal Decomposition and Coordination for Distributed Load Restoration in AC/DC Hybrid System. IEEE Transactions on Smart Grid, 12(2), 1685 - 1698. https://doi.org/10.1109/TSG.2020.3025694

\section{General rights}

Copyright and moral rights for the publications made accessible in the public portal are retained by the authors and/or other copyright owners and it is a condition of accessing publications that users recognise and abide by the legal requirements associated with these rights.

- Users may download and print one copy of any publication from the public portal for the purpose of private study or research.

- You may not further distribute the material or use it for any profit-making activity or commercial gain

- You may freely distribute the URL identifying the publication in the public portal 


\title{
Spatio-Temporal Decomposition and Coordina- tion for Distributed Load Restoration in AC/DC Hy- brid System
}

\author{
Jin Zhao, Student Member, IEEE, Hongtao Wang, Senior Member, IEEE, Qiuwei Wu, Senior Mem-
} ber, IEEE, Nikos D. Hatziargyriou, Fellow, IEEE, Wen Zhang, Member, IEEE

\begin{abstract}
A new spatio-temporal decomposition and coordination scheme is proposed for load restoration in an AC/DC hybrid transmission system. First, the framework of spatio-temporal decomposition is built for load restoration, the decoupled variables and spatial and temporal coordinators are defined, as well as two interactive blocks. Furthermore, the spatio-temporal decomposed models of subsystems and HVDC links are constructed considering security constraints and HVDC operation characteristics. Finally, a new hierarchical overlapping coordination based analytical target cascading (HOC_ATC) algorithm is developed to coordinate the decomposed models by iterative calculations between subsystems and coordinators, as well as the two interactive blocks. The proposed distributed scheme maintains independent operations of subsystems, and handles the multi-step restoration process by iteratively calculating single-step models. The developed HOC_ATC algorithm provides tractable and efficient computation for the HVDC model incorporated load restoration optimization. The effectiveness of the proposed method is validated using two IEEE test systems, showing improved computational efficiency and better convergence ability.

Index Terms - Analytical target cascading, hierarchical overlapping coordination, power system resilience, load restoration, AC/DC hybrid system.
\end{abstract}

\section{NOMENCLATURE}

\section{A. Indices}

$i \quad$ Index of nodes

$j, k \quad$ Indices of subsystems

$m \quad$ Indices of restoration step number

$L_{\mathrm{AB}}, L_{\mathrm{A}}, L_{\mathrm{B}}$ Indices of iteration between Blocks $\mathrm{A}$ and $\mathrm{B}$, iteration in Block A, and iteration in Block B

* Index for variables with fixed values

B. Sets

$N_{s u b} \quad$ Set of subsystems

$N_{\mathrm{L}, j} \quad$ Set of load nodes in subsystem $j$

$N_{\mathrm{B}, j} \quad$ Set of boundary nodes in subsystem $j$

$N_{\mathrm{B}, j, \mathrm{VSC}}, N_{\mathrm{B}, j, \mathrm{LCC}}, N_{\mathrm{B}, j, \mathrm{HVAC}}$

This work was supported by the Science and Technology Project of State Grid Corporation of China (Research on Key Sub-station Control Technology for Rapid Recovery of AC/DC Hybrid Power Grid) (Corresponding author: Hongtao Wang, Qiuwei Wu).

J. Zhao, H. Wang and W. Zhang are with the Key Laboratory of Power System Intelligent Dispatch and Control of Ministry of Education (Shandong University), Jinan 250000, China (e-mail: jinzhao_sdu@mail.sdu.edu.cn; whtwhm@sdu.edu.cn; zhangwen@sdu.edu.cn).

Q. Wu is with the Center for Electric Power and Energy, Department of Electrical Engineering, Technical University of Denmark, Kgs. Lyngby, DK 2800 (e-mail: qw@elektro.dtu.dk).

N. D. Hatziargyriou is with the National Technical University of Athens, Athens 15773, Greece (e-mail: nh@power.ece.ntua.gr).
Sets of boundary nodes of subsystem $j$ connected to VSC-HVDC, LCC-HVDC and HVAC

$N_{\mathrm{LCC}}, N_{\mathrm{VSC}}, N_{\mathrm{HVAC}}$

Sets of LCC-HVDC, VSC-HVDC and HVAC tie-lines.

\section{Parameters}

$T_{M} \quad$ Number of restoration steps

$\boldsymbol{c}_{\mathrm{L}, j}, \boldsymbol{p}_{\mathrm{L}, j}$ Vectors of load weighting coefficient and load amount in subsystem $j$,

$T_{f}, T_{l} \quad$ Parameters for multi-step coupled steps

$w_{j}, v_{j} \quad$ Lagrangian multipliers for subsystem $j$

$\beta \quad$ Updating parameter for Lagrangian multiplier

D. Variables

$\boldsymbol{x}_{\mathrm{L}, j, m}, \boldsymbol{p}_{\mathrm{G}, j, m}, \boldsymbol{S}_{\mathrm{B}, j, m}$

Vectors of load pickup decision variables, generator output variables and boundary variables in subsystem $j$ at time step $m$

$\boldsymbol{S}_{\mathrm{tie}, \mathrm{B}, j, m} \boldsymbol{P}_{\mathrm{tie}, m}, \chi_{\mathrm{tie}, m}$

Vectors of tie-line boundary variables related to subsystem $j$, active power through HVDC links and HVDC operation variables at time step $m$

$\boldsymbol{P}_{\text {tie }, j}^{\text {step }}, \boldsymbol{\chi}_{\text {tie }}^{\text {step }}$ Vectors of multi-step $\boldsymbol{P}_{\text {tie }, m}$ and $\chi_{\text {tie }, m}$

$\boldsymbol{S}_{\mathrm{B}, j, m}^{\mathrm{g}}, \boldsymbol{x}_{\mathrm{L}, \mathrm{g}, m}^{\mathrm{g}}, \boldsymbol{P}_{\mathrm{tie}}^{\mathrm{ste}, \mathrm{g}}, \chi_{\mathrm{tic}}^{\mathrm{sep}, \mathrm{g}}$

Vectors of auxiliary variables for coordinators

$\boldsymbol{\sigma}_{m} \quad$ Vectors of mismatch values of decoupled variables

$\boldsymbol{Y}_{a}, \boldsymbol{Y}_{b}$ Vectors of linking variables of blocks A and B

$x_{\mathrm{L}, j, m}^{g}, x_{\mathrm{L}, j, i, m, m-1}, x_{\mathrm{L}, j, i, m, m}$

Auxiliary load pickup variable of coordinator, step $m$ and step $m-1$ for node $i$ in subsystem $j$ at step $m$

$\sigma_{\mathrm{L}, j, i, m}$ Mismatch of load pickup variables for node $i$ in subsystem $j$ at step $m$

$P_{\mathrm{B}, j, i, m}, Q_{\mathrm{B}, j, i, m}, \theta_{\mathrm{B}, j, i, m}, V_{\mathrm{B}, j, i, m}$

Boundary variables of active power, reactive power, angle and voltage at boundary node $i$ of subsystem $j$ at step $m$

$P_{\mathrm{C} i, j, m}, Q_{\mathrm{C} i, j, m}, \theta_{\mathrm{C} i j, m}, V_{\mathrm{C} i, j, m}$

Variables of tie-line $C_{i}$ at step $m$

$P_{\mathrm{B}, j, i, m}^{\mathrm{g}}, Q_{\mathrm{B}, j, i, m}^{\mathrm{g}}, \theta_{\mathrm{B}, j, i, m}^{\mathrm{g}}, V_{\mathrm{B}, j, i, m}^{\mathrm{g}, \mathrm{s}}$

Auxiliary variables of coordinators

$P_{\mathrm{B}, \mathrm{AC}, \mathrm{R}, m}, Q_{\mathrm{B}, \mathrm{AC}, \mathrm{R}, m}, V_{\mathrm{AC}, \mathrm{R}, m}$

Boundary variables of $\mathrm{AC}$ power flow based active

power, reactive power and voltage on the HVDC rectifi-

er side at step $m$

$P_{\mathrm{B}, \mathrm{AC}, \mathrm{R}, m}, Q_{\mathrm{B}, \mathrm{AC}, \mathrm{R}, m}, V_{\mathrm{AC}, \mathrm{R}, m}$

Variables on the HVDC inverter side

$\sigma_{S u b, P, j, i, m}, \sigma_{S u b, Q, j, i, m}, \sigma_{S u b, \theta, j, i, m}, \sigma_{S u b, V, j, i, m}$

Boundary mismatch of active power, reactive power,

angle and voltage of subsystem $j$ at step $m$

$\sigma_{\mathrm{VSC}, P, C i, m}, \sigma_{\mathrm{VSC}, P, C i, m}$ 
Boundary mismatch of active power and reactive power of VSC-HVDC $C_{i}$ at step $m$

$\sigma_{\mathrm{LCC}, P, C i, m}, \sigma_{\mathrm{LCC}, Q, C i, m}, \sigma_{\mathrm{LCC}, V, C i, m}$

Boundary mismatch of active power, reactive power and voltage of LCC-HVDC $C_{i}$ at step $m$

$\sigma_{\mathrm{HVAC}, P, C i, m}, \sigma_{\mathrm{HVAC}, \theta, C i, m}$,

Boundary mismatch of active power and angle of

HVAC $C_{i}$ at step $m$

\section{INTRODUCTION}

$\mathrm{R}$ ESTORING a power system quickly after a large-area blackout is crucial for reducing social and economic costs [1]. In the restoration process, "build-up" is a common strategy for power system restoration [2]-[4]. In the build-up approach, the entire system is set up from small individual grids to speed up the whole system restoration process [5]. The build-up process conducts a parallel restoration and ends up with individual subsystems with energized networks and restarted generators [5], [6]. With limited generation and different load conditions, these independently operated subsystems should be reconnected and the un-served load needs to be restored to complete the whole restoration process [4]. This paper focuses on the load restoration of the last stage of the build-up process.

The strategies for transmission power system restoration are mainly based on AC power systems. However, in the 2016 and 2018 outages in Brazil, the failure of HVDC links led to irreparable power imbalance of subsystems and further induced system collapse [7]. Thus, the HVDC link has become an important part in the restoration process. A thorough introduction to show how HVDC links assist the power system restoration was provided in [8]. Ref. [9] presented the possibility of using HVDC links at the receiving end in the restoration process. Ref. [10] proposed a black-start optimization method utilizing HVDC links to accelerate restoration. A common condition for the $\mathrm{AD} / \mathrm{DC}$ hybrid bulk system is that several AC regional subsystems are connected by HVDC links or HVAC lines, e.g. the ultra-high-voltage (UHV) power system of China [11]. When a blackout occurs, HVDC links are locked, and consequently they become splitting points to form subsystems. Since both HVDC link technologies, linecommutated converter based HVDC (LCC-HVDC) and voltage-sourced converter based HVDC (VSC-HVDC) links, are able to be restarted in the black-start stage [8]-[10], they can be used for load restoration. An HVDC link can interconnect regional subsystems without the need of synchronization [8], which makes it convenient to reconnect isolated subsystems.

Considering the operation of HVDC links between subsystems, load restoration is performed in an AC/DC hybrid system. HVDC models are normally built and incorporated into the optimal power flow (OPF) model in order to optimize the control and operation of the AC/DC hybrid system [12]-[15]. These studies solve the optimization problem with a centralized approach, where all the information needs to be sent to the control center. However, restoration of subsystems is implemented independently [5]-[6], which means that complete information may not be available during this restoration pro- cess. Moreover, the AC/DC system has several subsystems divided by HVDC links. This system feature makes it suitable to solve the optimal operation problem in a distributed manner [16]. Therefore, a distributed approach, which allows subsystems to operate independently and be coordinated only based on boundary information, is preferred for the load restoration of the AC/DC hybrid system.

Distributed algorithms are normally divided into Lagrangian relaxation based methods, such as the analytical target cascading (ATC), alternating direction method of multipliers (ADMM) and the auxiliary problem principle, and the Karush-Kuhn-Tucker conditions based methods, such as the optimality condition decomposition and heterogeneous decomposition [17]. Since the ATC method is a model-based distributed method and does not require convexity of the model, it is convenient to incorporate HVDC models. Ref. [18]-[19] propose ATC based voltage control methods, which are very helpful for dispatching large-scale wind farm clusters. In [16] a three-level hierarchical scheme based on the ATC method is proposed, in order to realize decentralized optimal operation of the AC/DC hybrid distribution system. Ref. [20] used ATC to perform distributed unit commitment in the AC/DC hybrid transmission system. The distributed optimization of the AC/DC hybrid systems in [16] and [20] focus on spatial decomposition. Load restoration, however, is a temporally coupled process with coupled multi-step decision variables and coupled operation conditions of HVDC links in a sequence of time periods [20]. The multi-step situation and highly nonlinear HVDC models [16] result in a largely extended model scale and increased complexity. Therefore, temporal decomposition is also needed to maintain the models with single-step scale. In general, the subsystem load restoration in the AC/DC hybrid system is a spatial-temporal coupled multi-step problem and needs spatial-temporal decomposition to achieve tractable computation. The binary variables, highly non-linear HVDC models and large number of coupling variables in the spatio-temporal models may lead to non-convergence of the ATC method based iterative calculation. Modification of the ATC method is needed to ensure its feasibility for the load restoration problem of the $\mathrm{AC} / \mathrm{DC}$ system.

In this paper, a new spatio-temporal decomposition and coordination method is proposed for distributed load restoration in the $\mathrm{AC} / \mathrm{DC}$ hybrid transmission system. The hierarchical overlapping coordination based ATC (HOC_ATC) algorithm is developed to obtain the final distributed load restoration strategy. First, the structure of the spatio-temporal decomposition and coordination is designed composed of two interacting blocks. Then, the detailed spatio-temporal decomposed models are constructed considering restoration security constraints and operation characteristics of HVDC links. Finally, the HOC_ATC method is applied to solve and coordinate the spatio-temporal decomposed models by iterative calculation. The distributed spatio-temporal solution is obtained when the iterative calculation process converges.

The contributions of this paper are as follows: 1) A new distributed load restoration scheme is proposed to complete the build-up process of an $\mathrm{AD} / \mathrm{DC}$ hybrid bulk system. It provides 
distributed load restoration strategy for the large-scale complex system with several AC regional subsystems connected by HVDC links and HVAC lines. 2) The spatial-temporal decomposition and coordination with two interactive blocks is designed. Accordingly, the multi-step load restoration model incorporated with HVDC links is transformed into computationally tractable forms. The spatial decomposition achieves independent decision-making of each subsystem, and the temporal decomposition further reduces the scale of the optimization models. 3) The HOC_ATC algorithm is developed to solve the spatio-temporal models by iterative calculation. As an improved distributed method, it reduces the model scale in iterative calculation and shows better convergence ability compared with the standard ATC algorithm.

The rest of the paper is organized as follows: Section II describes the framework of spatial-temporal decomposition and coordination of the AC/DC system load restoration model. The detailed spatial-temporal decomposed models are provided in Section III. Section IV presents the proposed HOC_ATC algorithm. Section V demonstrates the efficacy of the proposed method by case studies, followed by conclusions.

\section{Framework of SPATIO-TEMPORALly DeCOMPOSED AND COORDINATED LOAD RESTORATION IN AC/DC HYBRID SYSTEM}

\section{A. Load restoration of subsystems in AC/DC hybrid system}

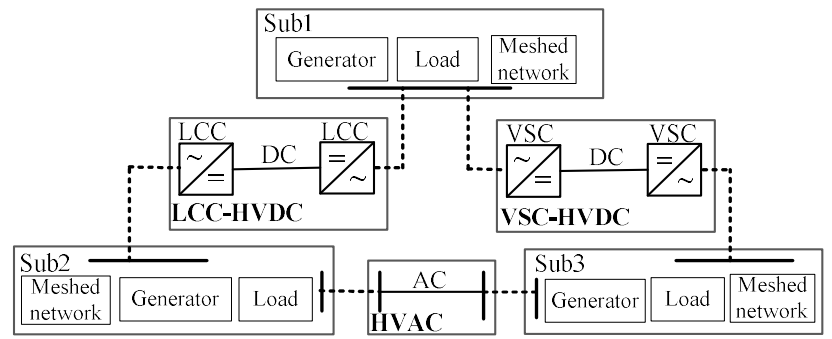

Fig. 1. Structure of the AC/DC system with individual subsystems.

During the last stage of the build-up restoration process, several individual subsystems typically exist. Transmission lines within these subsystems are energized, generators are ramping up and a large amount of loads remains un-served [5], [6]. As shown in Fig. 1, regional grids are connected by tielines. In an AC/DC hybrid system, tie-lines are distinguished in HVAC tie-lines and HVDC links. Depending on converter technologies, HVDC links include LCC-HVDC and VSCHVDC [9]. The LCC-HVDC is widely used, while VSCHVDC has higher flexibility in terms of control and operation.

Isolated subsystems need to be reconnected to form the interconnected system and the remaining un-served loads should be picked up to complete the restoration process [4]. The restoration process is a multi-step process. Therefore, the restoration optimization model needs to be built as a step-by-step optimization [17] or multi-step optimization [21], [22]. Considering the spatial coupling of reconnected subsystems and the temporal coupling of different restoration steps, the multistep load restoration model for the whole AC/DC hybrid system is model (1) in a compact form.

In model (1), $\boldsymbol{g}_{j, m}()$ and $\boldsymbol{h}_{j, m}()$ are the inequality and equality constraints including load pickup constraints, generator output limits, $\mathrm{AC}$ power flow constraints and voltage and frequency constraints of subsystem $j$ at step $m$, and $\mathbf{g}_{j k, m}()$ and $\boldsymbol{h}_{j k, m}()$ are the constraints for HVDC links and HVAC tie-lines including steady-state power flow equations and control limits of converters. $\boldsymbol{f}_{j, \mathrm{~m}}()$ represents multi-step coupled constraints of load pickup and boundary variables for subsystem $j . \boldsymbol{f}_{j k . \mathrm{m}}()$ includes the multi-step coupled constraints for power flow reversion and power adjustment of HVDC links between subsystem $j$ and $k . \boldsymbol{P}_{\mathrm{tie}}^{\mathrm{step}}=\left[\boldsymbol{P}_{\mathrm{tie}, m-T f}, \ldots, \boldsymbol{P}_{\mathrm{tie}, m}, \ldots, \boldsymbol{P}_{\mathrm{tie}, m+T l}\right]$ and $\chi_{\mathrm{tie}}^{\mathrm{step}}=\left[\chi_{\mathrm{tie}, m-T f}, \ldots\right.$, $\left.\chi_{\mathrm{tie}, m}, \ldots, \chi_{\mathrm{tie}, m+T]}\right]$ refer to the tie-line power and control variables for HVDC/HVAC tie-lines in the multi-step process. The objective is to maximize the weighted load recovery amount in all subsystems during the multi-step process. The detailed model corresponding to model (1) is provided in Section III. $A$.

$$
\begin{array}{ll}
\min & \sum_{m=1}^{T_{M}} \sum_{j \in N_{\mathrm{Sub}}}-\boldsymbol{c}_{\mathrm{L}, j} \boldsymbol{p}_{\mathrm{L}, j} \boldsymbol{x}_{\mathrm{L}, j, m} \\
\text { s.t. } & \boldsymbol{g}_{j, m}\left(\boldsymbol{x}_{\mathrm{L}, j, m}, \boldsymbol{p}_{\mathrm{G}, j, m}, \boldsymbol{S}_{\mathrm{B}, j, m}\right) \leq 0 \\
& \boldsymbol{h}_{j, m}\left(\boldsymbol{x}_{\mathrm{L}, j, m}, \boldsymbol{p}_{\mathrm{G}, j, m}, \boldsymbol{S}_{\mathrm{B}, j, m}\right)=0 \\
& \boldsymbol{f}_{j, m}\left(\boldsymbol{x}_{\mathrm{L}, j, m}, \boldsymbol{x}_{\mathrm{L}, j, m-1}, \boldsymbol{S}_{\mathrm{B}, j, m}, \boldsymbol{S}_{\mathrm{B}, j, m-1}\right) \leq 0 \\
& \boldsymbol{g}_{j k, m}\left(\boldsymbol{S}_{\mathrm{tie}, \mathrm{B}, j, m}, \boldsymbol{S}_{\mathrm{tie}, \mathrm{B}, k, m}, \boldsymbol{P}_{\mathrm{tie}, m}, \chi_{\mathrm{tie}, m}\right) \leq 0 \\
& \boldsymbol{h}_{j k, m}\left(\boldsymbol{S}_{\mathrm{tie}, \mathrm{B}, j, m}, \boldsymbol{S}_{\mathrm{tie}, \mathrm{B}, k, m}, \boldsymbol{P}_{\mathrm{tie}, m}, \boldsymbol{\chi}_{\mathrm{tie}, m}\right)=0 \\
& \boldsymbol{f}_{j k, m}\left(\boldsymbol{P}_{\mathrm{tie}}^{\mathrm{step}}, \boldsymbol{\chi}_{\mathrm{tie}}^{\mathrm{step}}\right) \leq 0 \\
& \sigma_{m}=\left[\boldsymbol{S}_{\mathrm{B}, j, m}-\boldsymbol{S}_{\mathrm{B}, j, m}^{\mathrm{g}}, \boldsymbol{S}_{\mathrm{tie}, \mathrm{B}, j, m}-\boldsymbol{S}_{\mathrm{B}, j, m}^{\mathrm{g}}, \boldsymbol{x}_{\mathrm{L}, j, m}\right. \\
& \left.-\boldsymbol{x}_{\mathrm{L}, j, m}^{\mathrm{g}}, \boldsymbol{P}_{\mathrm{tie}}^{\mathrm{step}}-\boldsymbol{P}_{\mathrm{tie}}^{\mathrm{ste}, \mathrm{g}}, \chi_{\mathrm{tie}}^{\text {step }}-\chi_{\mathrm{tie}}^{\mathrm{step}, \mathrm{g}}\right]=0 \\
& \forall m \in\left[1, \ldots, T_{M}\right], \forall j, k \in N_{\mathrm{Sub}}, j \neq k
\end{array}
$$

\section{B. Spatio-temporal decomposition and coordination}

Because of the coupled spatial and temporal variables in $\boldsymbol{\sigma}_{m}$, the load restoration problem for the $\mathrm{AC} / \mathrm{DC}$ system is modelled in (1) in a centralized way. However, the model may not be feasible, because the subsystems are normally operated independently. In spatial terms, due to commercial or security requirements, the information of each subsystem cannot be completely available to form the centralized optimization model. Temporally, centralized multi-step optimization may be computationally intractable, especially for large-scale systems whose model scale is largely extended with variables and constraints of several steps. A distributed form with spatiotemporal decomposition is therefore much more suitable to solve the multi-step load restoration problem of an AC/DC hybrid system.

In order to realize a distributed form, coupled spatial and temporal variables in $\boldsymbol{\sigma}_{m}$ can be decoupled using the Lagrangian decomposition method [23]. Each constraint set $\boldsymbol{\sigma}_{m}=0$ can be transformed into a quadratic penalty function $\Phi\left(\boldsymbol{\sigma}_{m}\right)$ added in the objective function to relax constraints [24]. Accordingly, the spatio-temporal decomposition is achieved.

$$
\Phi\left(\sigma_{m}\right)=\boldsymbol{v} \sigma_{m}{ }^{\mathrm{T}}+\left\|\boldsymbol{w} \circ \boldsymbol{\sigma}_{m}{ }^{\mathrm{T}}\right\|_{2}^{2}
$$

where $\boldsymbol{v}$ and $\boldsymbol{w}$ are vectors of penalty multipliers for model decoupling. The symbol $\circ$ represents the Hadamard product which is an entry-wise multiplication of two vectors. 
BLOCK A

Sp patial-decomposition

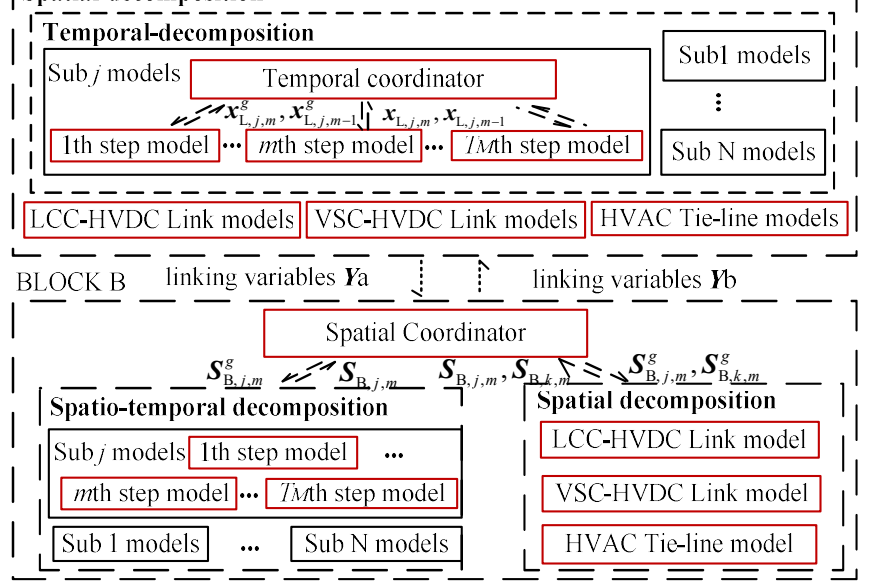

Fig. 2. Structure of the spatio-temporal decomposition and coordination

The direct spatio-temporal decomposition of the model is very difficult due to the many coupling variables in $\boldsymbol{\sigma}_{m}$. Since many constraints are relaxed, it is hard to guarantee the consistency of the decoupled variables. Therefore, the HOC method [25] is used in the decomposition process to reduce the number of coupled variables. Fig. 2 shows the framework of the proposed spatio-temporal decomposition. Firstly, the model is separated into two blocks A and B. The two blocks exchange linking variables with each other. By defining the linking variables between the two blocks as (3)-(4), the coupled variables can be largely reduced. In block $\mathrm{A}$ which has a nature spatial decomposition using fixed boundary variables in $\boldsymbol{Y}_{b}$, the temporal coordinator is formed to transform the multistep model into single-step models. In block B with a nature temporal decomposition using fixed multi-step variables in $\boldsymbol{Y}_{a}$, the spatial coordinator is built to divide the whole system into subsystems with links between them.

$$
\begin{aligned}
& \left\{\begin{array}{l}
\boldsymbol{Y}_{a}=\left[\boldsymbol{x}_{\mathrm{L}}^{\text {step }}\right]=\left[\boldsymbol{x}_{\mathrm{L}, 1}^{\text {step }}, \ldots, \boldsymbol{x}_{\mathrm{L}, j}^{\text {step }}, \ldots, \boldsymbol{x}_{\mathrm{L}, \mathrm{J}}^{\text {step }}\right] \\
\boldsymbol{x}_{\mathrm{L}, j}^{\text {step }}=\left[\boldsymbol{x}_{\mathrm{L}, j, 1}, \ldots, \boldsymbol{x}_{\mathrm{L}, j, m}, \ldots, \boldsymbol{x}_{\mathrm{L}, j, T_{M}}\right]
\end{array}\right. \\
& \left\{\begin{array}{l}
\boldsymbol{Y}_{b}=\left[\boldsymbol{S}_{\mathrm{B}}^{\text {step }}\right]=\left[\boldsymbol{S}_{\mathrm{B}, 1}^{\text {step }}, \ldots, \boldsymbol{S}_{\mathrm{B}, j}^{\text {step }}, \ldots, \boldsymbol{S}_{\mathrm{B}, J}^{\text {step }}\right] \\
\boldsymbol{S}_{\mathrm{B}, j}^{\text {step }}=\left[\boldsymbol{S}_{\mathrm{B}, j, 1}, \ldots, \boldsymbol{S}_{\mathrm{L}, j, m}, \ldots, \boldsymbol{S}_{\mathrm{L}, j, T_{M}}\right]
\end{array}\right.
\end{aligned}
$$

In a distributed way, the spatio-temporal decomposition is coordinated according to interactions in Fig. 2. Three types of coordination exist in the spatio-temporal decomposition framework. The coordination between the two blocks is based on the interaction of linking variables, the spatial coordination depends on the boundary variable information and the temporal coordination relies on the multi-step load pickup information. The spatial decomposition achieves independent decision-making of each subsystem, and the temporal decomposition further reduces the optimization model scale. The detailed models used in Fig. 2 are provided in Section III and the HOC_ATC algorithm is presented in Section IV.

\section{SPATIO-TEMPORALLY DECOMPOSED MODELS FOR LOAD RESTORATION IN AN AC/DC HYBRID SYSTEM}

In this section the distributed models with spatio-temporal decomposition are constructed. First, the original multi-step load restoration model considering security constraints and operation characteristics of HVDC links is presented. Then, the spatio-temporal decomposed models in Block A and Block $\mathrm{B}$ are described.

\section{A. Load restoration model in AC/DC hybrid system}

The original model, which corresponds to Model (1), consists of the objective function, VSC-HVDC link model [16], the LCC-HVDC link model [14], [26] and subsystem load restoration models [27]. The detailed models are presented in Appendix A.

The non-linear steady-state power flow equations of HVDC links are used to represent converters. The non-linear model is widely used in $\mathrm{AD} / \mathrm{DC}$ system planning [28], operation [16], [29] and control optimization [30], because it reveals the operation characteristic of HVDC links. The non-linear equations consider detailed HVDC control variables, which ensures that the obtained restoration strategy can be implemented according to the control strategy of HVDC converters. As such, the feasibility of the distributed restoration method is ensured in practical AC/DC hybrid systems.

Linearized $\mathrm{AC}$ power flow calculations are integrated into subsystem load restoration models. Although DC power flow is a popular technique to handle the distributed transmission system optimization problem [18], [31], [32], the AC power flow formulation is selected to provide the voltage and active/reactive power at boundary buses related to steady-state power flow of HVDC links. The linearization is based on the linear programming approximation of AC power flow (LPAC) method in [33] which approximates original non-linear $\mathrm{AC}$ power flow equations using a series of linear constraints. Ref. [33]-[34] justify the exactness of the LPAC model for the power system restoration, and the LPAC method has been successfully used in the load restoration problem [22].

\section{B. Models of Block $A$}

As linking variables transmitted from Block B, the shared boundary variables $\boldsymbol{S}_{\mathrm{B}, j}^{*}$ in Block A have fixed values. For the VSC-HVDC connected boundary, LCC-HVDC connected boundary and HVAC connected one, are $\boldsymbol{S}_{\mathrm{B}, j}^{*}=\left[\boldsymbol{P}_{\mathrm{B}, j}^{*}, \boldsymbol{Q}_{\mathrm{B}, j}^{*}\right], \boldsymbol{S}_{\mathrm{B}, j}^{*}=[\boldsymbol{P}$ $\left.{ }_{\mathrm{B}, j}^{*}, \boldsymbol{Q}_{\mathrm{B}, j}^{*}, \boldsymbol{V}_{\mathrm{B}, j}^{*}\right]$ and $\boldsymbol{S}_{\mathrm{B}, j}^{*}=\left[\boldsymbol{P}_{\mathrm{B}, j}^{*}, \boldsymbol{\theta}_{\mathrm{B}, j}^{*}\right]$, respectively. Since boundary variables are fixed, the models in Block A are spatially decomposed, which means subsystems, HVAC tie-lines and HVDC links can manage their operation models independently.

1) Spatio-temporally decomposed subsystem model. In order to further reduce the model complexity due to temporal coupling, each subsystem model performs temporal decomposition by relaxing the constraints for a sequence of time periods. By introducing $\left[x_{\mathrm{L}, j, i, m}^{g}, x_{\mathrm{L}, j, i, m, m-1}, x_{\mathrm{L}, j, i, m, m}\right]$ which satisfy (5), multi-step coupled load pickup decision variables $x_{\mathrm{L}, j, i, m}$ and $x_{\mathrm{L}, j, i, m-}$ 1 in each subsystem are replaced by the decoupled $x_{\mathrm{L}, j, i, m, m-1}$, $x_{\mathrm{L}, j, i, m, m}$ using the Lagrangian decomposition method [24].

$$
\begin{gathered}
\sigma_{\mathrm{L}, j, i, m}=x_{\mathrm{L}, j, i, m}^{\mathrm{g}}-x_{\mathrm{L}, j, i, m, m} \& \sigma_{\mathrm{L}, j, i, m-1}=x_{\mathrm{L}, j, i, m-1}^{\mathrm{g}}-x_{\mathrm{L}, j, i, m, m-1} \\
\forall i \in N_{\mathrm{L}, j}, \forall m \in\left\{1, \ldots, T_{M}\right\} \\
\min \sum_{m=1}^{T_{M}} \sum_{i \in N_{\mathrm{L}, j}}\left(v_{j} \sigma_{\mathrm{L}, j, i, m}+\left\|w_{j} \sigma_{\mathrm{L}, j, i, m}\right\|_{2}^{2}\right)+\sum_{m=2 i \in N_{\mathrm{L}, j}}^{T_{M}}\left(v_{j} \sigma_{\mathrm{L}, j, i, m-1}+\left\|w_{j} \sigma_{\mathrm{L} j, i, m-1}\right\|_{2}^{2}\right) \\
j \in N_{\mathrm{Sub}}, \forall i \in N_{\mathrm{L}, j}, \forall m \in\left\{1, \ldots, T_{M}\right\}
\end{gathered}
$$


Each subsystem contains one temporal coordinator model (6) which coordinates its spatio-temporally decomposed model (7).

$$
\begin{aligned}
& \min -\sum_{i \in N_{\mathrm{L}, j}} c_{\mathrm{L}, j, i, m} p_{\mathrm{L}, j, i, m} x_{\mathrm{L}, j, i, m}+\sum_{i \in N_{\mathrm{L}, j}}\left(\begin{array}{l}
v_{j} \sigma_{\mathrm{L}, j, i, m}+\left\|w_{j} \sigma_{\mathrm{L}, j, i, m}\right\|_{2}^{2} \\
v_{j} \sigma_{\mathrm{L}, j, i, m-1}+\left\|w_{j} \sigma_{\mathrm{L}, j, i, m-1}\right\|_{2}^{2}
\end{array}\right) \\
& \text { s.t. }(\mathrm{A}-36)-(\mathrm{A}-54) \\
& \quad P_{\mathrm{B}, j, i, m}=P_{\mathrm{B}, j, i, m}^{*}, Q_{\mathrm{B}, j, i, m}=Q_{\mathrm{B}, j, i, m}^{*}\left(i \in N_{\mathrm{B}, j, \mathrm{VSC}}\right) \\
& P_{\mathrm{B}, j, i, m}=P_{\mathrm{B}, j, i, m}^{*}, Q_{\mathrm{B}, j, i, m}=Q_{\mathrm{B}, j, i, m}^{*}, V_{\mathrm{B}, j, i, m}=V_{\mathrm{B}, j, i, m}^{*}\left(i \in N_{\mathrm{B}, j, \mathrm{LCC}}\right) \\
& \quad P_{\mathrm{B}, j, i, m}=P_{\mathrm{B}, j, i, m}^{*}, \theta_{\mathrm{B}, j, i, m}=\theta_{\mathrm{B}, j, i, m}^{*}\left(i \in N_{\mathrm{B}, j, \mathrm{HVAC}}\right) \\
& \quad j \in N_{\mathrm{Sub}}, \forall m \in\left\{1, \ldots, T_{M}\right\}
\end{aligned}
$$

2) Spatially decomposed models of links between subsystems. Constraints (A-34)-(A-35) for HVAC tie-lines are satisfied with feasible boundary variables $\left[\boldsymbol{P}_{\mathrm{B}, j, m}^{*}, \boldsymbol{\theta}_{\mathrm{B}, \mathrm{j}, \mathrm{m}}^{*}\right]$. Spatially decomposed models of VSC-HVDC and LCC-HVDC links satisfy (8)-(9), respectively.

$$
\begin{aligned}
& E . q .(\mathrm{A}-2)-(\mathrm{A}-14),(\mathrm{A}-29)-(\mathrm{A}-31) \\
& P_{\mathrm{B}, \mathrm{AC}, \mathrm{R}, m}=P_{\mathrm{B}, j, i, m}^{*}, Q_{\mathrm{B}, \mathrm{AC}, \mathrm{R}, m}=Q_{\mathrm{B}, j, i, m}^{*}\left(i \in N_{\mathrm{B}, j, \mathrm{VSC}}\right) \\
& P_{\mathrm{B}, \mathrm{AC}, \mathrm{I}, m}=P_{\mathrm{B}, k, i, m}^{*}, Q_{\mathrm{B}, \mathrm{AC}, \mathrm{L}, m}=Q_{\mathrm{B}, k, i, m}^{*}\left(i \in N_{\mathrm{B}, k, \mathrm{VSC}}\right) \\
& \forall m \in\left\{1, \ldots, T_{M}\right\}
\end{aligned}
$$

E.q. (A-15)-(A-28),(A-29)-(A-33)

$$
\begin{aligned}
& P_{\mathrm{B}, \mathrm{AC}, \mathrm{R}, m}=P_{\mathrm{B}, j, i, m}^{*}, Q_{\mathrm{B}, \mathrm{AC}, \mathrm{R}, m}=Q_{\mathrm{B}, j, i, m}^{*}, V_{\mathrm{ACR}, m}=V_{\mathrm{B}, j, i, m}^{*}\left(i \in N_{\mathrm{B}, j, \mathrm{LCC}}\right) \\
& P_{\mathrm{B}, \mathrm{AC}, \mathrm{l}, m}=P_{\mathrm{B}, k, i, m}^{*}, Q_{\mathrm{B}, \mathrm{AC}, \mathrm{l}, m}=Q_{\mathrm{B}, k, i, m}^{*}, V_{\mathrm{AC}, \mathrm{I}, m}=V_{\mathrm{B}, k, i, m}^{*}\left(i \in N_{\mathrm{B}, k, \mathrm{LCC}}\right) \\
& \forall m \in\left\{1, \ldots, T_{M}\right\}
\end{aligned}
$$

\section{Models of Block B}

The models in Block $B$ have fixed values of load pickup decision variables $\boldsymbol{x}_{\mathrm{L}, \mathrm{s}}^{\text {stp }}$ which are the linking variables transmitted from Block A. There is a spatial coordinator for the whole system in Block B to realize the independent decisionmaking of each subsystem. Because the multi-step coupled load pickup variables are fixed, subsystem models can be spatio-temporally decomposed when the boundary variables in $\boldsymbol{S}_{\mathrm{B}, j, m}$ are decoupled. Extra variables $\left[P_{\mathrm{B}, j, i, m}^{g}, Q_{\mathrm{B}, j, i, m}^{g}, \theta_{\mathrm{B}, j, i, m}^{g}, V_{\mathrm{B}_{j, j, m}}^{g}\right]$, $\left[P_{\mathrm{B}, j, i, m, m-1}, P_{\mathrm{B}, j, i, m, m}, \theta_{\mathrm{B}, j, i, m}, Q_{\mathrm{B}, j, i, m}, V_{\mathrm{B}, j, i, m}\right],\left[P_{\mathrm{C} i, j, m}, \theta_{\mathrm{C} i, j, m}, P_{\mathrm{C} i, k, m}\right.$, $\left.\theta_{\mathrm{C} i, k, m}\right],\left[P_{\mathrm{C} i j, j, m}, Q_{\mathrm{C} i j, m}, P_{\mathrm{C} i, k, m}, Q_{\mathrm{C} i, k, m}\right]$ and $\left[P_{\mathrm{C} i j, j, m}, \theta_{\mathrm{C} i j, m}, V_{\mathrm{C} i j, j, m}\right.$, $\left.P_{\mathrm{C} i, k, m}, \theta_{\mathrm{C} i, k, m}, V_{\mathrm{C} i, k, m}\right]$ are introduced for the spatial coordinator, subsystems, HVAC tie-lines, VSC-HVDC links and LCCHVDC links, respectively. The coupling constraints (10)-(13) are required as the extension of (A-55) - (A-57).

$$
\begin{aligned}
& \sigma_{S u b, P, j, i, m}=P_{\mathrm{B}, j, i, m}^{\mathrm{g}}-P_{\mathrm{B}, j, i, m, m}=0\left(i \in N_{\mathrm{B}, j}\right) \\
& \sigma_{S u b, P, j, i, m-1}=P_{\mathrm{B}, j, i, m-1}^{\mathrm{g}}-P_{\mathrm{B}, j, i, m, m-1}=0\left(i \in N_{\mathrm{B}, j}\right) \\
& \sigma_{S u b, Q, j, i, m}=Q_{\mathrm{B}, j, i, m}^{\mathrm{g}}-Q_{\mathrm{B}, j, i, m}=0 \quad\left(i \in N_{\mathrm{B}, j, \mathrm{LCC}} \& N_{\mathrm{B}, j, \mathrm{VSC}}\right) \\
& \sigma_{\text {Sub, }, j, j, m}=\theta_{\mathrm{B}, j, i, m}^{\mathrm{g}}-\theta_{\mathrm{B}, j, i, m}=0 \quad\left(i \in N_{\mathrm{B}, j, \mathrm{HVAC}}\right) \\
& \sigma_{\text {Sub },, j, i, m}=V_{\mathrm{B}, j, i, m}^{\mathrm{g}}-V_{\mathrm{B}, j, i, m}=0 \quad\left(i \in N_{\mathrm{B}, j, \mathrm{LCC}}\right) \\
& \forall j \in N_{\mathrm{Sub}}, \forall m \in\left\{1, \ldots, T_{M}\right\}
\end{aligned}
$$

$$
\begin{array}{r}
\sigma_{\mathrm{VSC}, P, C_{i}, m}=P_{\mathrm{B}, j, i, m}^{\mathrm{g}}-P_{\mathrm{B}, \mathrm{AC}, C_{i}, m}=0\left(i \in N_{\mathrm{B}, j, \mathrm{VSC}}, C_{i} \in N_{\mathrm{VSC}}\right) \\
\sigma_{\mathrm{VSC}, Q, C_{i}, m}=Q_{\mathrm{B}, j, i, m}^{\mathrm{g}}-Q_{\mathrm{B}, \mathrm{AC}, C_{i}, m}=0\left(i \in N_{\mathrm{B}, j, \mathrm{VSC}}, C_{i} \in N_{\mathrm{VSC}}\right) \\
\forall j \in N_{\mathrm{Sub}}, \forall m \in\left\{1, \ldots, T_{M}\right\} \\
\sigma_{\mathrm{LCC}, P, C_{i}, m}=P_{\mathrm{B}, j, i, m}^{\mathrm{g}}-P_{\mathrm{B}, \mathrm{AC}, C_{i}, m}=0\left(i \in N_{\mathrm{B}, j, \mathrm{LCC}}, C_{i} \in N_{\mathrm{LCC}}\right) \\
\sigma_{\mathrm{LCC}, Q, C_{i}, m}=Q_{\mathrm{B}, j, i, m}^{\mathrm{g}}-Q_{\mathrm{B},, \mathrm{AC}, C_{i}, m}=0\left(i \in N_{\mathrm{B}, j, \mathrm{LCC}}, C_{i} \in N_{\mathrm{LCC}}\right) \\
\sigma_{\mathrm{LCC}, V, C_{i}, m}=V_{\mathrm{B}, j, i, m}^{\mathrm{g}}-V_{\mathrm{B}, \mathrm{AC}, C_{i}, m}=0\left(i \in N_{\mathrm{B}, j, \mathrm{LCC}}, C_{i} \in N_{\mathrm{LCC}}\right) \\
\forall j \in N_{\mathrm{Sub}}, \forall m \in\left\{1, \ldots, T_{M}\right\} \\
\sigma_{\mathrm{HVAC}, P, C_{i}, m}=P_{\mathrm{B}, j, i, m}^{\mathrm{g}}-P_{\mathrm{B}, A C, C_{i}, m}=0\left(i \in N_{\mathrm{B}, j, \mathrm{HVAC}}, C_{i} \in N_{\mathrm{HVAC}}\right) \\
\sigma_{\mathrm{HVAC}, \theta, C_{i}, m}=\theta_{\mathrm{B}, j, i, m}^{\mathrm{g}}-\theta_{\mathrm{B}, \mathrm{AC}, C_{i}, m}=0\left(i \in N_{\mathrm{B}, j, \mathrm{HVAC}}, C_{i} \in N_{\mathrm{HVAC}}\right) \\
\forall j \in N_{\mathrm{Sub}}, \forall m \in\left\{1, \ldots, T_{M}\right\}
\end{array}
$$

1) Model of spatial coordinator. The spatial coordinator model (14) is built to coordinate the decoupled boundary variables by relaxing (10)-(13) using (2). The first three terms are constructed for the consistency of subsystem boundary variables, and the last three terms are made for the consistency of boundary variables of VSC-HVDC links, LCC-HVDC links and HVAC tie-lines, respectively.

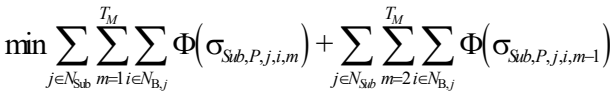

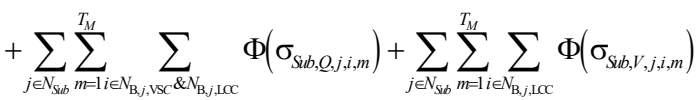

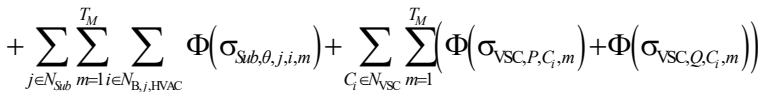

$$
\begin{aligned}
& +\sum_{C \in N_{\mathrm{LCC}}} \sum_{m=1}^{T_{M}}\left(\Phi\left(\sigma_{\mathrm{LOC}, P, C, m}\right)+\Phi\left(\sigma_{\mathrm{LCC}, Q, C, m}\right)+\Phi\left(\sigma_{\mathrm{LCC}, V, C, m}\right)\right) \\
& +\sum_{C \in N_{\mathrm{HNC}}} \sum_{m=1}^{T_{\mathrm{H}}}\left(\Phi\left(\sigma_{\mathrm{HNAC}, P, C, m}\right)+\Phi\left(\sigma_{\mathrm{HNAC}, \theta, C_{i}, m}\right)\right)
\end{aligned}
$$

2) Spatio-temporally decomposed subsystem model. With fixed load pickup decision variables in $\boldsymbol{x}_{\mathrm{L}, \mathrm{j}, m}^{*}$, the spatiotemporally decomposed subsystem model is (15).

$$
\begin{aligned}
\min & \sum_{i \in N_{\mathrm{B}, j}}\left(\Phi\left(\sigma_{S u b, P, j, i, m}\right)+\Phi\left(\sigma_{S u b, P, j, i, m-1}\right)\right) \\
& +\sum_{i \in N_{\mathrm{B}, j, \mathrm{HV} A C}} \Phi\left(\sigma_{S u b, \theta, j, i, m}\right)+\sum_{i \in N_{\mathrm{B}, j, \mathrm{YSC}}} \Phi\left(\sigma_{S u b, Q, j, i, m}\right) \\
& +\sum_{i \in N_{\mathrm{B}, j, \mathrm{LCC}}}\left(\Phi\left(\sigma_{S u b, Q, j, i, m}\right)+\Phi\left(\sigma_{S u b, V, j, i, m}\right)\right) \\
\text { s.t. } & (\mathrm{A}-37)-(\mathrm{A}-54) \\
& \boldsymbol{x}_{\mathrm{L}, j, i, m}=\boldsymbol{x}_{\mathrm{L}, j, i, m}^{*}, \boldsymbol{x}_{\mathrm{L}, j, i, m-1}=\boldsymbol{x}_{\mathrm{L}, j, i, m-1}^{*}\left(\forall i \in N_{\mathrm{L}, j}\right)
\end{aligned}
$$

3) Spatial decomposed models of links between subsystems. Model (16)-(18) are built for VSC-HVDC links, LCC-HVDC links and HVAC tie-lines, respectively.

$$
\begin{array}{ll}
\min & \sum_{m=1}^{T_{M}}\left(\Phi\left(\sigma_{\mathrm{VSC}, P, C_{i}, m}\right)+\Phi\left(\sigma_{\mathrm{vSC}, Q, C_{i}, m}\right)\right) \\
\text { s.t. } & (\mathrm{A}-2)-(\mathrm{A}-14),(\mathrm{A}-29)-(\mathrm{A}-31) \\
& C_{i} \in N_{\mathrm{VSC}}
\end{array}
$$




$$
\begin{aligned}
& \min \sum_{m=1}^{T_{M}}\left(\Phi\left(\sigma_{\mathrm{LCC}, P, C_{i}, m}\right)+\Phi\left(\sigma_{\mathrm{LCC}, Q, C_{i}, m}\right)+\Phi\left(\sigma_{\mathrm{LCC}, V, C_{i}, m}\right)\right) \\
& \text { s.t. }(\mathrm{A}-15)-(\mathrm{A}-28),(\mathrm{A}-29)-(\mathrm{A}-33) \\
& C_{i} \in N_{\mathrm{LCC}} \\
& \min \sum_{m=1}^{T_{M}}\left(\Phi\left(\sigma_{\mathrm{HVAC}, P, C_{i}, m}\right)+\Phi\left(\sigma_{\mathrm{HVAC}, \theta, C_{i}, m}\right)\right) C_{i} \in N_{\mathrm{HVAC}}
\end{aligned}
$$

\section{Solution Method Based on HOC ATC Algorithm}

This section develops the HOC-ATC algorithm to completely decompose and solve the spatial-temporal models of Section III. The information interaction and iterative calculation process are presented. The distributed load restoration strategy is obtained when the iterative calculation process converges.

\section{A. Iterative calculation based on HOC_ATC algorithm}

The HOC_ATC based calculation process is shown below.

Step 0: Set $L_{\mathrm{AB}}=1$ and set convergence thresholds $\varepsilon_{1}$ and $\varepsilon_{2}$ for Block B, $\varepsilon_{3}$ and $\varepsilon_{4}$ for Block $\mathrm{A}$ and $\varepsilon_{5}$ between the two blocks equal to acceptable small values. Choose any feasible initial values for load pickup decision variables $\boldsymbol{X}_{\mathrm{L}}^{*}$ in $\boldsymbol{Y}_{\mathrm{a}}^{\mathrm{L}^{\wedge B}}$ for each restoration step of all the subsystems.

Step 1: Set $L_{\mathrm{B}}=1$ and define the initial values of the penalty multipliers in $\boldsymbol{v}^{L_{B}}$ and $\boldsymbol{w}^{L_{\mathrm{B}}}$. Choose any feasible initial values for global boundary variables $\boldsymbol{S}_{\mathrm{B}}^{\mathrm{g}, L \mathrm{AB}, L \mathrm{~B}}=\boldsymbol{S}_{\mathrm{B}}^{\mathrm{g} *}$ for each subsystem and links between subsystems.

Step 2: Solve subsystem models (15), VSC-HVDC model (16), LCC-HVDC model (17) and HVAC tie-line model (18) with linking variables in $\boldsymbol{Y}_{\mathrm{a}}^{\mathrm{AB}_{\mathrm{B}}}$ and global boundary variables in $\boldsymbol{S}_{\mathrm{B}}^{\mathrm{g}, L_{\mathrm{AB}}, L_{\mathrm{B}}}$ in parallel, and obtain local boundary variables $\boldsymbol{S}_{\mathrm{B}}^{L_{\mathrm{AB}}, L_{\mathrm{B}}}=$ $\boldsymbol{S}_{\mathrm{B}}^{*}$ and objective values of all the models as $\boldsymbol{f}^{\mathrm{AB}, L \mathrm{~B}}$.

Step 3: Solve the spatial coordinator model (14) with fixed $\boldsymbol{S}_{\mathrm{B}}^{L \mathrm{AB}, L \mathrm{~B}}$ and obtain the global boundary variables $\boldsymbol{S}_{\mathrm{B}}^{\mathrm{g}, L \mathrm{LB}, L \mathrm{~B}+1}=\boldsymbol{S}_{\mathrm{B}}^{\mathrm{g}^{*}}$.

Step 4: Check the spatial coordinator guided convergence equations (19)-(21), if they are satisfied, go to Step 6, otherwise, go to Step 5.

$$
\begin{gathered}
\left|\boldsymbol{S}_{\mathrm{B}, i}^{g, L_{\mathrm{AB}}, L_{\mathrm{B}}+1}-\boldsymbol{S}_{\mathrm{B}, i}^{L_{\mathrm{AB}}, L_{\mathrm{B}}}\right| \leq \varepsilon_{3} \forall i \in N_{\mathrm{Sub}}, N_{\mathrm{VSC}}, N_{\mathrm{LCC}}, N_{\mathrm{HVAC}} \\
\left|\left(f_{i}^{L_{\mathrm{AB}}, L_{\mathrm{B}}}-f_{i}^{L_{\mathrm{AB}}, L_{\mathrm{B}}-1}\right) / f_{i}^{L_{\mathrm{AB}}, L_{\mathrm{B}}}\right| \\
L_{\mathrm{B}} \geq \varepsilon_{4} \forall i \in N_{\mathrm{Bub}}, N_{\mathrm{VSC}}, N_{\mathrm{LCC}}, N_{\mathrm{HVAC}}
\end{gathered}
$$

Step 5: Update penalty parameters $\boldsymbol{v}^{L_{\mathrm{B}}}$ and $\boldsymbol{w}^{L_{\mathrm{B}}}$ according to (22) and (23), set $L_{\mathrm{B}}=L_{\mathrm{B}}+1$ and go back to Step 2 .

$$
\begin{gathered}
\boldsymbol{v}_{i}^{L_{\mathrm{B}}+1}=\boldsymbol{v}_{i}^{L_{\mathrm{B}}}+2\left(\boldsymbol{w}_{i}^{L_{\mathrm{B}}}\right) Q\left(\boldsymbol{w}_{i}^{L_{\mathrm{B}}}\right)^{\mathrm{T}}\left(\boldsymbol{S}_{\mathrm{B}, i}^{g, L_{\mathrm{B}}, L_{\mathrm{B}}+1}-\boldsymbol{S}_{\mathrm{B}, i}^{L_{\mathrm{BB}}, L_{\mathrm{B}}}\right) \\
\forall i \in N_{\mathrm{Sub}}, N_{\mathrm{VSC}}, N_{\mathrm{LCC}}, N_{\mathrm{HVAC}} \\
\boldsymbol{w}_{i}^{L_{\mathrm{B}}+1}=\boldsymbol{\beta} \boldsymbol{w}_{i}^{L_{\mathrm{B}}}(\beta \geq 1) \forall i \in N_{\mathrm{Sub}}, N_{\mathrm{VSC}}, N_{\mathrm{LCC}}, N_{\mathrm{HVAC}}
\end{gathered}
$$

Step 6: Collect the optimal result in the $L_{\mathrm{B}}$-th iteration and form linking variables $\boldsymbol{Y}_{\mathrm{b}}^{L_{B}}=\boldsymbol{S}_{\mathrm{B}}^{L_{\mathrm{B}}, L_{B}+1}$, set $L_{\mathrm{A}}=1$ and define the initial values of the penalty multipliers in $\boldsymbol{v}^{L_{\Lambda}}$ and $\boldsymbol{w}^{L_{\Lambda}}$, and choose initial values for local load pickup decision variables $\boldsymbol{x}$ ${ }_{\mathrm{L}}^{g, L B, L A}=\boldsymbol{x}_{\mathrm{L}}^{\boldsymbol{x}^{*}}$ in each restoration step of all the subsystems.

Step 7: Solve models (7) of each subsystem with linking variables in $\boldsymbol{Y}_{\mathrm{b}}^{L_{\mathrm{B}}}$ and global load pickup decision variables in $\boldsymbol{x}$ ${ }_{\mathrm{L}}^{\mathrm{g}, L \mathrm{AB}, L \Lambda}$ in parallel, and obtain local load pickup decision varia- bles $\boldsymbol{x}_{\mathrm{L}}^{L_{\mathrm{AB}}, L_{A}}=\boldsymbol{x}_{\mathrm{L}}^{*}$ and objective values of subsystems as $\boldsymbol{f}^{\mathrm{AB}_{\mathrm{B}}, L_{A}}$.

Step 8: Solve models of temporal coordinators (14) of subsystems with fixed $\boldsymbol{x}_{\mathrm{L}}^{L_{\mathrm{LB}}, L_{\Lambda}}$ in parallel, solve equations of HVDC links (8)-(9) if $L_{\mathrm{A}}=1$, and obtain the global load pickup decision variables $\boldsymbol{x}_{\mathrm{L}}^{\mathrm{g}, L \Delta \mathrm{LB}, L_{\Lambda}+1}=\boldsymbol{x}_{\mathrm{L}}^{\mathrm{g}^{*}}$ and decision variables for HVDC links $\chi_{\text {tic }}^{L_{\mathrm{BB}}}=\chi_{\text {tic }}^{*}$.

Step 9: Check the temporal coordinator guided convergence equations (24)-(26), if they are satisfied, proceed to Step 11, otherwise, go to Step 10.

$$
\begin{gathered}
\left|x_{L, j, m}^{g, L_{\mathrm{AB}}, L_{\mathrm{A}}+1}-x_{L, j, m}^{L_{\mathrm{AB}}, L_{\mathrm{A}}}\right| \leq \varepsilon_{1} \forall j \in N_{\mathrm{Sub}}, m \in\left[1, \ldots, T_{M}\right] \\
\left|\left(f_{j}^{L_{\mathrm{AB}}, L_{\mathrm{A}}}-f_{j}^{L_{\mathrm{AB}}, L_{\mathrm{A}}-1}\right) / f_{j}^{L_{\mathrm{AB}}, L_{\mathrm{A}}}\right| \leq \varepsilon_{2} \quad \forall j \in N_{\mathrm{Sub}} \\
L_{\mathrm{A}} \geq L_{\mathrm{A}, \max }
\end{gathered}
$$

Step 10: Update penalty parameters $\boldsymbol{v}^{L_{\Lambda}}$ and $\boldsymbol{w}^{L_{\Lambda}}$ according to (35) and (36), set $L_{\mathrm{A}}=L_{\mathrm{A}}+1$, and go back to Step 7 .

$$
\begin{gathered}
\boldsymbol{v}_{j}^{L_{\mathrm{A}}+1}=\boldsymbol{v}_{j}^{L_{\mathrm{A}}}+2\left(\boldsymbol{w}_{j}^{L_{\mathrm{A}}}\right) Q\left(\boldsymbol{w}_{j}^{L_{\mathrm{A}}}\right)^{\mathrm{T}}\left(\boldsymbol{x}_{L, j}^{g, L_{\mathrm{A}}, L_{\mathrm{A}}+1}-\boldsymbol{x}_{L, j}^{L_{\mathrm{A}}, L_{\mathrm{A}}}\right) \quad \forall j \in N_{\text {Sub }} \\
\boldsymbol{w}_{j}^{L_{\mathrm{A}}+1}=\beta \boldsymbol{w}_{j}^{L_{\mathrm{A}}}(\beta \geq 1) \quad \forall j \in N_{\text {Sub }}
\end{gathered}
$$

Step 11: Collect the optimal result in the $L_{\mathrm{A}}$-th iteration and form linking variables $\boldsymbol{Y}_{\mathrm{a}}^{\mathrm{LAB}}=\boldsymbol{x}_{\mathrm{L}}^{L_{\mathrm{B}, L \Lambda+1}}$, and check the convergence conditions for the two blocks (29)-(30), if they are not satisfied, $L_{\mathrm{AB}}=L_{\mathrm{AB}}+1$ and go back to Step 1 , otherwise, stop and obtain the final result.

$$
\begin{gathered}
\boldsymbol{Y}_{\mathrm{a}}^{L_{\mathrm{AB}}}=\boldsymbol{Y}_{\mathrm{a}}^{L_{\mathrm{AB}}-1} \&\left|\boldsymbol{Y}_{\mathrm{b}}^{L_{\mathrm{AB}}}-\boldsymbol{Y}_{\mathrm{b}}^{L_{\mathrm{AB}}-1}\right| \leq \boldsymbol{\varepsilon}_{5} \\
L_{\mathrm{AB}} \leq L_{\mathrm{AB}, \max }
\end{gathered}
$$

In this algorithm, Steps 1 - 5 concern the iterative calculation in Block B, Steps 7 - 10 takes the one in Block A and Step 11 manages the iteration between the two blocks.

\section{B. Analysis of HOC_ATC based iterative calculation}

As shown in Fig. 3, there are three types of iterative calculations in the proposed HOC_ATC based solution process.

The first type of iterative calculation is performed in Block $\mathrm{B}$ with fixed load pickup decision variables $\boldsymbol{x}_{\mathrm{L}}^{\text {step }}$. Each subsystem independently optimizes its own spatio-temporally decomposed Model (15), and is linked with the other subsystems to solve optimization Models (16)-(18). Then, the local boundary variables $\boldsymbol{S}_{\mathrm{B}}^{*}$ are sent to the spatial coordinator. Holding local boundary variables, the spatial coordinator solves Model (14) and sends global boundary variables $\boldsymbol{S}_{\mathrm{B}}^{\mathrm{e}^{*}}$ to subsystems, HVDC link and HVAC tie-line models. The calculation in Block B is completed when the spatial coordinator guided iteration process converges.

The second type of iterative calculation is implemented within Block A with fixed boundary variables $\boldsymbol{S}_{\mathrm{B}}^{*}$. In each subsystem, local load pickup decisions $\boldsymbol{x}_{\mathrm{L}, \mathrm{j}}^{*}$ are sent to the temporal coordinator when the single-step model (7) is solved. By solving model (6), each temporal coordinator transmits global load pickup decisions $\boldsymbol{x}_{\mathrm{L}, j}^{\mathrm{g}^{*}}$ to the corresponding subsystem model. Calculations within Block A are completed when the temporal coordinator guided iterations in all the subsystems converge and HVDC link models (8)-(9) are solved.

The third type of iteration is between Block A and Block B, Block A sends linking variables $\boldsymbol{Y}_{\mathrm{a}}^{*}=\boldsymbol{x}_{\mathrm{L}}^{\text {ste }}$ to Block B and Block B sends linking variables $\boldsymbol{Y}_{\mathrm{b}}^{*}=\boldsymbol{S}_{\mathrm{B}}^{*}$ to Block A. Both blocks per- 
form calculations with part of decision variables fixed. The iterative calculation between Block A and Block B continues until the convergence conditions are satisfied.

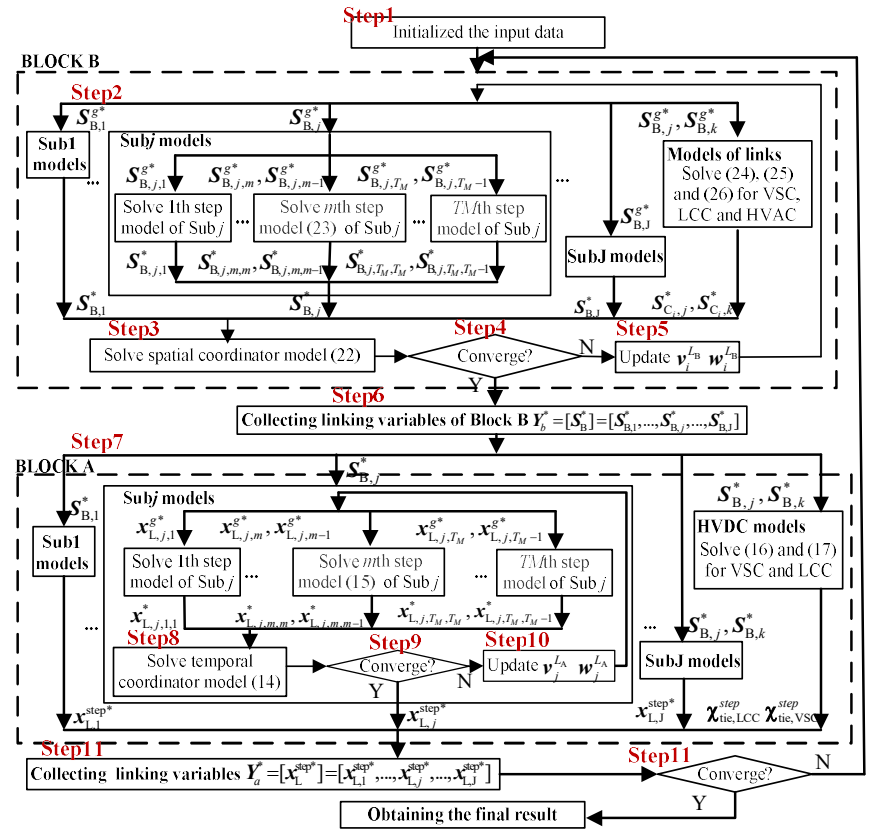

Fig. 3 Flowchart of iterative calculation of HOC_ATC algorithm

Although it is difficult to give theoretically global convergence proof when models have highly non-convex parts and binary variables, the proposed HOC_ATC algorithm has good convergence qualities. The iterative calculation within Block A and Block B are based on the ATC method, which can converge to the result with a small optimization gap, even when the model is non-convex [16]-[17], [20]. Moreover, the HOC based iteration between two blocks ensures that the result of each iteration gradually gets close to the optimal result [25]. In Block A, the temporal coordinator model (6) and subsystem model (7) have a mixed-integer quadratic programming (MIQP) form, and HVDC link models (8)-(9) comprise several non-linear equations. In Block $\mathrm{B}$, the spatial coordinator model (14) and subsystem model (15) are quadratic programming (QP) problems, and HVDC link models (16)-(17) are non-linear optimization problems without integer variables. The MIQP and QP models are of small scale and the number of non-linear equations of HVDC links models is also small, so that they can be efficiently solved. Detailed convergence analysis is in Appendix B.

\section{CASE Study}

Two systems were used to demonstrate the effectiveness of the proposed method. The first one is a modified three interconnected 6-bus AC/DC hybrid system [20]. The second one is a sectionalized restoration IEEE 118-bus system. The convergence thresholds of the HOC_ATC algorithm are $0.1 \%$.

\section{A. Efficiency of spatio-temporal decomposition and} coordination scheme in 6-bus system

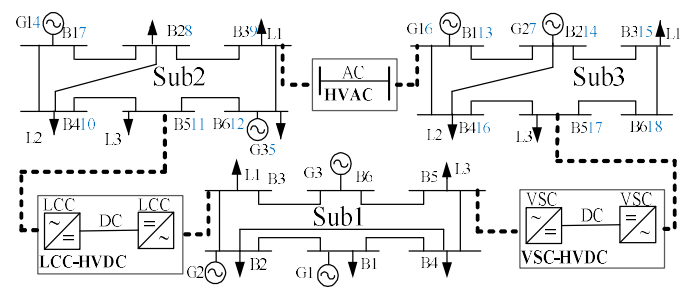

Fig. 4. Three-area 18-bus AC/DC hybrid system

Fig. 4 shows the three-area 18-bus AC/DC hybrid system. The multi-step number is set as three. Although subsystem models are linearized, the highly non-convex HVDC models make the whole problem hard to solve efficiently in a centralized way. As shown in Table I, the spatio-temporal coupled model for the 18-bus system can be directly solved using Fmincon. The calculation time is $2966.70 \mathrm{~s}$ and the objective value is -9.50 p. u.. Based on the HOC_ATC algorithm, the subsystem model and HVDC model can be solved independently. With QP in Block A and MIQP in Block B, subsystem models are iteratively solved using Gurobi, while HVDC models are solved using Fmincon. The proposed HOC_ATC method takes $33.32 \mathrm{~s}$ to find the objective value $-13.50 \mathrm{p}$. u., exhibiting faster computation and better optimization result than the centralized one.

TABLE I

COMPUTATION EFFICIENCY OF DiFfERENT METHOD

\begin{tabular}{|c|c|c|c|c|}
\hline & Obj (p. u.) & Slover & Cal_time (s) & Iteration \\
\hline Centralized & -9.50 & Fmincon & 2966.70 & 1 \\
\hline HOC_ATC & -13.50 & $\begin{array}{l}\text { Gurobi+ } \\
\text { Fmincon }\end{array}$ & 33.32 & 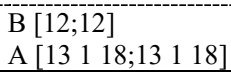 \\
\hline
\end{tabular}

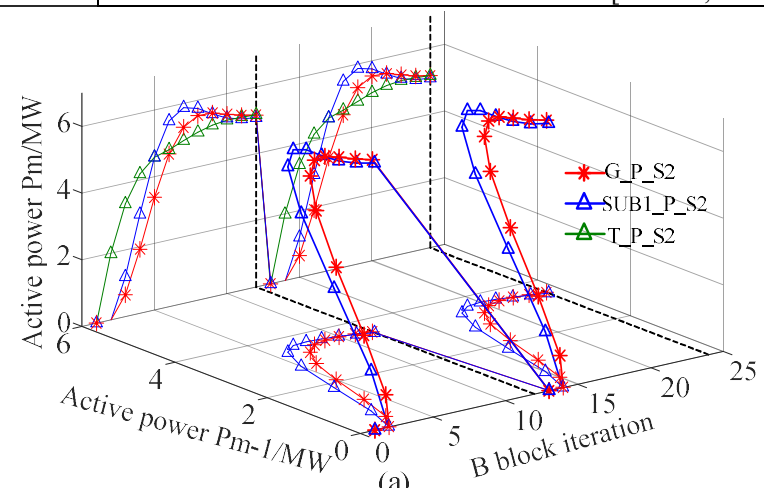

(a)

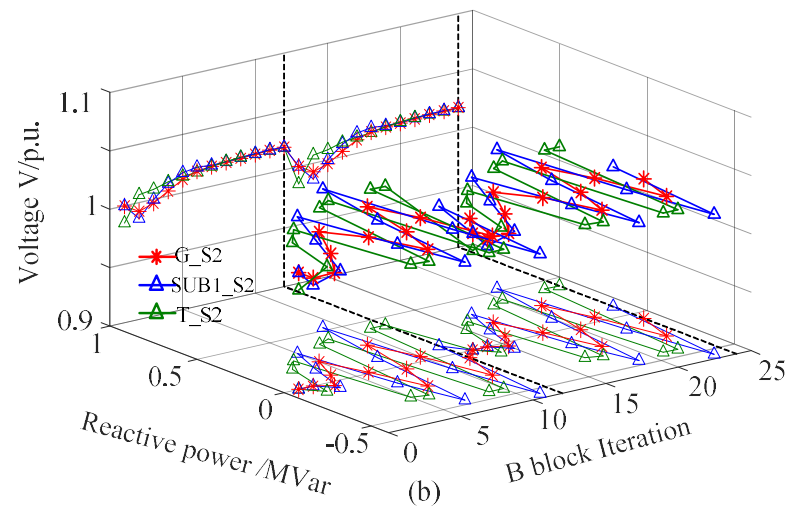

Fig. 5. Active power (a), reactive power and voltage (b) iterations of SUB2 step 2 in Block B 


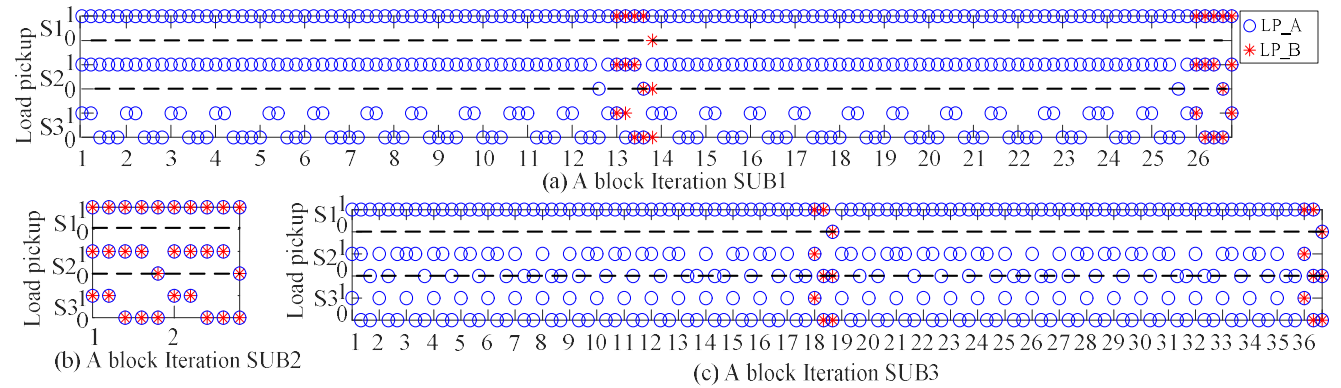

Fig. 6. Iterations of load pickup decisions in Block A

Using the HOC ATC algorithm, the model is decomposed into spatial-temporally decoupled single-step subsystem optimization models and spatially decoupled HVDC link models. The whole process starts with the iterative calculations in Block B. Taking boundary variables between SUB1 and the LCC-HVDC link in Step 2 as an example, Fig. 5 shows the iteration process of shared boundary variables. In Fig. 5 (a), 'G_P_S2', 'SUB1_P_S2' and T_P_S2 refer to boundary active power variables of the spatial coordinator, subsystem and LCC-HVDC links, respectively. The subsystem active power on the x-z plane is the spatially decoupled variable for the optimization model of Step 2, while the one on the x-y plane is the temporally decoupled variable of Step 2 for the optimization model of Step 3. In Fig. 5 (b), the x, y and $\mathrm{z}$ axes are the iteration number, voltage value and reactive power, respectively. It can be seen that all the boundary variables of the spatial coordinator, the subsystem side and the HVDC side satisfy convergence thresholds after the Block B iteration.

Then, the calculation goes to Block A. Fig. 6 shows the iteration of load pickup decisions of three subsystems in three restoration Steps S1, S2 and S3. The iteration numbers of SUB1, SUB2 and SUB3 are 13, 1 and 18. The load pickup decisions 'LP_B' in Block $B$ are different from the result 'LP_A' obtained in Block A, which means load pickup decisions are adjusted in the iterations of Block A. Thus, the second round of Block B iteration begins with the result of Block A. Similarly, the calculation in Block B converges after 12 iterations and the second round of Block A calculation ends when all the iteration calculations within subsystems converge. Since the load pickup decision obtained from Block A is the same as in the last round, the whole HOC_ATC loop stops with the optimization result obtained according to the final round of Block A iteration.

\section{B. Computation efficiency of HOC_ATC method in IEEE 118-bus system}

The IEEE 118-bus system is divided into five restoration subsystems which can balance power supply and demand by themselves. Fig. 7 shows the structure of the system which uses the LCC-HVDC link, VSC-HVDC link and HVAC tielines connecting SUB1 and SUB2, SUB3 and SUB5 and the other subsystems. The centralized model for this case cannot be directly solved by general optimization tools due to its large-scale, highly non-convex form. Therefore, the HVDC incorporated large-scale model is solved in a distributed way.

The ATC based distributed method used in the AC/DC system [16], [20] is applied for comparison with the proposed HOC-ATC method. Convergence processes of different meth- ods are demonstrated in Fig. 8. As can be seen, the largest boundary variable mismatch of the HOC_ATC method is lower than $0.1 \%$ with 73 iterations, while the ATC method cannot converge with a mismatch higher than $1 \%$. Both methods perform iterative calculation with spatial-temporal decoupled models. However, the ATC method coordinates all the shared variables at the same time, while the HOC_ATC algorithm divides the original problem into two blocks and coordinates the corresponding variables separately. The convergence problem of the ATC method is caused by integer variables and the increase of shared variables in the multi-step process. In spatial-temporally decoupled models, the integer variables include not only load pickup decisions for the current step, but also the ones coming from temporal decoupling. Moreover, the ATC method coordinates integer variables and boundary variables simultaneously, which makes it hard to converge.

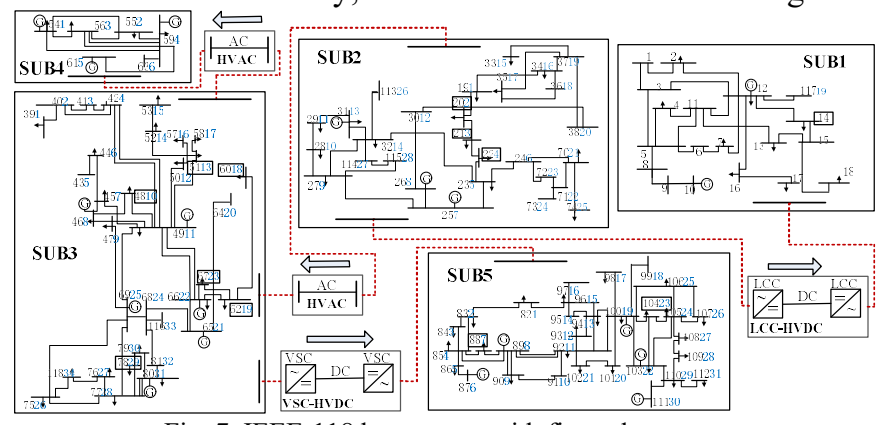

Fig. 7. IEEE-118 bus system with five subsystems

The iteration of the HOC_ATC method is shown in Fig. 9 using boundary active power variables of SUB1 'P', HVDC link ' $T$ P $P$ ' and the spatial coordinator ' $G$ _P' in five restoration steps $\mathrm{S} 1, \mathrm{~S} 2, \mathrm{~S} 3, \mathrm{~S} 4$ and $\mathrm{S} 5$. 'P21', 'P32', 'P43' and 'P54' are temporally decoupled active power variables of S1 S2 S3 and S4 for optimization models of S2 S3 S4 and S5. The first, second and third rounds of Block B iteration numbers are 17, 22 and 16. Iteration numbers in Block A for SUB1, SUB2, SUB3, SUB4 and SUB5 are 3, 3, 17, 4 and 14, respectively.

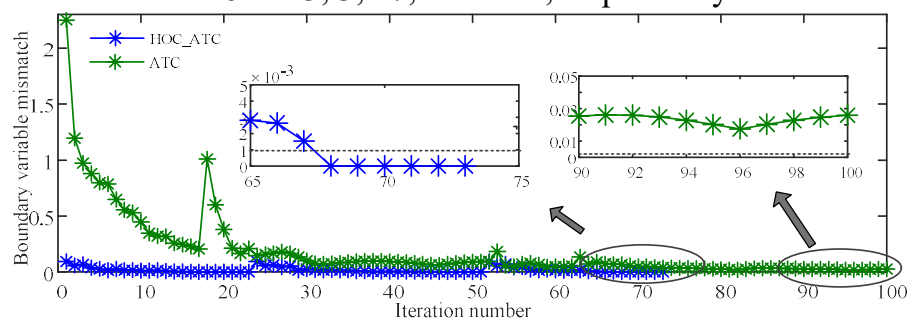

Fig. 8. Convergence processes of different methods. 


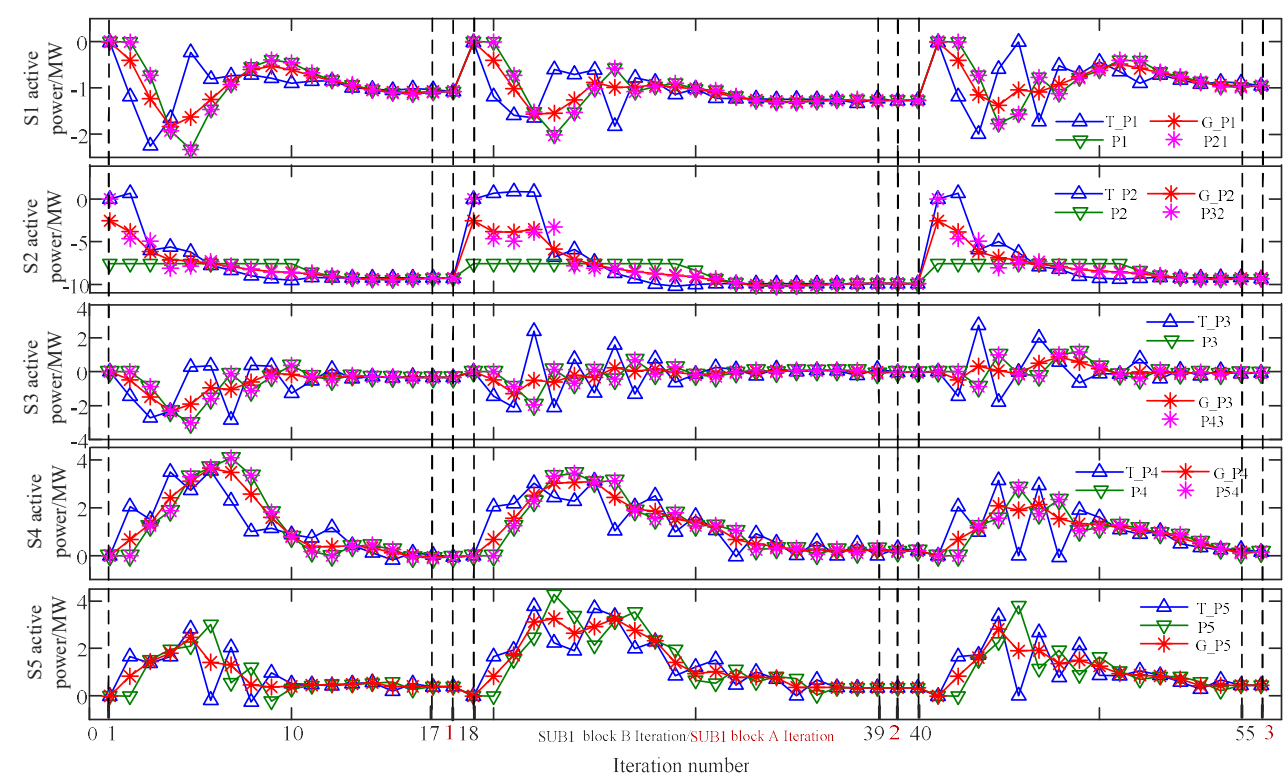

Fig. 9. Boundary variable values in the whole iteration process.

As seen in Fig. 10, the objective value changes according to the iteration process. The objective value changes in Block B because of the coordination process of boundary shared variables of all the subsystems, tie-line utilities and the spatial coordinator. In Block A, the change of objective value is caused by the coordination of load pickup decision variables using subsystem temporal coordinators. After 73 iterations, the final result is obtained at $-172.13 \mathrm{p}$. u.

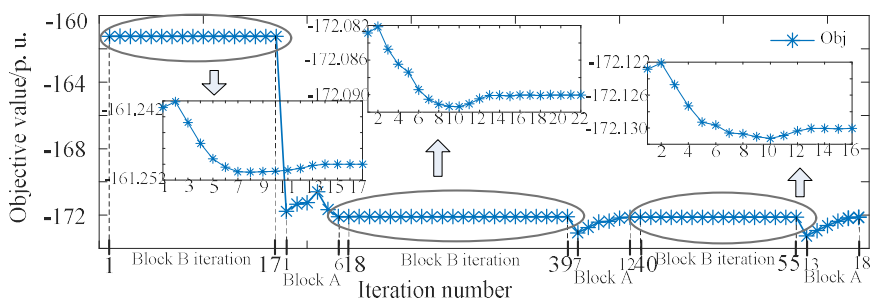

Fig. 10. The objective value in the whole iterative process.

\section{CONCLUSION}

This paper presents a new spatio-temporal decomposition and coordination scheme for load restoration in an $\mathrm{AC} / \mathrm{DC}$ hybrid transmission system. An improved distributed algorithm is proposed to realize the scheme. Using the proposed spatio-temporal decomposition method, the original HVDC incorporated multi-step model of the whole system is decomposed to a single-step load restoration model of each subsystem and operation model of each HVDC link. By applying the developed HOC_ATC algorithm, the result is obtained by iteratively calculating parallel small-scale models with simple mathematical forms within and between two interactive blocks. The proposed scheme maintains the independent operation of subsystems and provides a computationally tractable form of the highly complex load restoration model in the AC/DC hybrid bulk system. The case study shows the calculation process and the computation efficiency of the proposed method indicating computation efficiency and enhanced convergence abilities when facing highly non-convex multi-step models.
Acknowledgement

The authors would like to thank the editor and reviewers for their insightful comments and constructive suggestions that have substantially helped to improve the quality of this paper.

\section{Appendix A}

The detailed AC/DC bulk system load restoration model corresponds to Model (1).

1) Objective: The goal is restoring the un-served load to complete the restoration process as soon as possible. Therefore, the objective function $(\mathrm{A}-1)$ is to maximize the total load recovery amount of all the subsystems in the multi-step process. $c_{\mathrm{L}, j, i}$ and $p_{\mathrm{L}, j, i}$ are load weighting coefficient and load amount of load node $i$ in subsystem $j . x_{\mathrm{L}, j, i, m}$ is the load pick up binary decision variable for load node $i$ in subsystem $j$ in step $m$.

$$
\min -\sum_{m=1}^{T_{M}} \sum_{j \in S u b s y s} \sum_{i \in N_{\mathrm{L}, j}} c_{\mathrm{L}, j, i} p_{\mathrm{L}, j, i} x_{\mathrm{L}, j, i, m}
$$

2) Model of VSC-HVDC link: The steady state equations describing the two-terminal VSC-HVDC link are constructed as (A-2)-(A-9) [16].

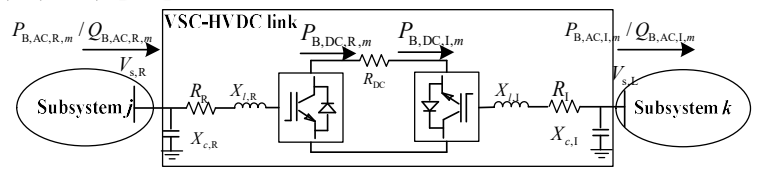

Fig. A-1 The equivalent circuit of the VSC-HVDC link

$$
\begin{aligned}
& P_{\mathrm{B}, \mathrm{AC}, \mathrm{R}, m}=\frac{\mu_{\mathrm{R}} M_{\mathrm{R}, m}}{\sqrt{2}} V_{\mathrm{s}, \mathrm{R}} V_{\mathrm{DC}, \mathrm{R}, m} Y_{\mathrm{R}} \sin \left(\delta_{\mathrm{R}, m}-\alpha_{\mathrm{R}}\right)+V_{\mathrm{s}, \mathrm{R}}^{2} Y_{\mathrm{R}} \sin \alpha_{\mathrm{R}}(\mathrm{A}-2) \\
& P_{\mathrm{B}, \mathrm{AC}, \mathrm{I}, m}=\frac{\mu_{\mathrm{I}} M_{\mathrm{I}, m}}{\sqrt{2}} V_{\mathrm{s}, \mathrm{I}} V_{\mathrm{DC}, \mathrm{I}, m} Y_{\mathrm{I}} \sin \left(\delta_{\mathrm{I}, m}-\alpha_{\mathrm{I}}\right)+V_{\mathrm{s}, \mathrm{I}}^{2} Y_{\mathrm{I}} \sin \alpha_{\mathrm{I}}(\mathrm{A}-3) \\
& Q_{\mathrm{B}, \mathrm{AC}, \mathrm{R}, m}=-\frac{\mu_{\mathrm{R}} M_{\mathrm{R}, m}}{\sqrt{2}} V_{\mathrm{s}, \mathrm{R}} V_{\mathrm{DC}, \mathrm{R}, m} Y_{\mathrm{R}} \cos \left(\delta_{\mathrm{R}, m}-\alpha_{\mathrm{R}}\right)+V_{\mathrm{s}, \mathrm{R}}^{2} Y_{\mathrm{R}} \cos \alpha_{\mathrm{R}}+\frac{V_{\mathrm{s}, \mathrm{R}}^{2}}{X_{C, \mathrm{R}}}(\mathrm{A}-4) \\
& Q_{\mathrm{B}, \mathrm{AC}, \mathrm{I}, m}=-\frac{\mu_{\mathrm{I}} M_{\mathrm{I}, m}}{\sqrt{2}} V_{\mathrm{s}, \mathrm{I}} V_{\mathrm{DC}, \mathrm{I}, m} Y_{\mathrm{I}} \cos \left(\delta_{\mathrm{I}, m}-\alpha_{\mathrm{I}}\right)+V_{\mathrm{s}, \mathrm{I}}^{2} Y_{\mathrm{I}} \cos \alpha_{\mathrm{I}}+\frac{V_{\mathrm{s}, \mathrm{I}}^{2}}{X_{C, \mathrm{I}}}(\mathrm{A}-5)
\end{aligned}
$$




$$
\begin{aligned}
P_{\mathrm{DC}, \mathrm{R}, m}= & \frac{\mu_{\mathrm{R}} M_{\mathrm{R}, m}}{\sqrt{2}} V_{\mathrm{S}, \mathrm{R}} V_{\mathrm{DC}, \mathrm{R}, m} Y_{\mathrm{R}} \sin \left(\delta_{\mathrm{R}, m}+\alpha_{\mathrm{R}}\right)-\frac{\mu_{\mathrm{R}}^{2} M_{\mathrm{R}}^{2}}{2} V_{\mathrm{DC}, \mathrm{R}, m}^{2} Y_{\mathrm{R}} \sin \left(\alpha_{\mathrm{R}}\right)(\mathrm{A}-6) \\
P_{\mathrm{DC}, \mathrm{I}, m} & =\frac{\mu_{\mathrm{I}} M_{\mathrm{I}, m}}{\sqrt{2}} V_{\mathrm{S}, \mathrm{I}} V_{\mathrm{DC}, \mathrm{I}, m} Y_{\mathrm{I}} \sin \left(\delta_{\mathrm{I}, m}+\alpha_{\mathrm{I}}\right)-\frac{\mu_{1}^{2} M_{\mathrm{I}}^{2}}{2} V_{\mathrm{DC}, \mathrm{I}, m}^{2} Y_{\mathrm{I}} \sin \left(\alpha_{\mathrm{I}}\right)(\mathrm{A}-
\end{aligned}
$$$$
P_{\mathrm{DC}, \mathrm{R}, m}=\left(V_{\mathrm{DC}, \mathrm{R}, m}-V_{\mathrm{DC}, \mathrm{I}, m}\right) / R_{\mathrm{DC}} * V_{\mathrm{DC}, \mathrm{R}, m}
$$$$
P_{\mathrm{DC}, \mathrm{I}, m}=\left(V_{\mathrm{DC}, \mathrm{R}, m}-V_{\mathrm{DC}, \mathrm{I}, m}\right) / R_{\mathrm{DC}} * V_{\mathrm{DC}, \mathrm{I}, m}
$$

where $P_{\mathrm{B}, \mathrm{AC}, \mathrm{R}, m}, Q_{\mathrm{B}, \mathrm{AC}, \mathrm{R}, m}, P_{\mathrm{DC}, \mathrm{R}, m}$ and $V_{\mathrm{DC}, \mathrm{R}, m}$ are the active power and reactive power from the subsystem, the DC active power flow and DC voltage on the rectifier side of the VSCHVDC link in step $m, P_{\mathrm{B}, \mathrm{AC}, \mathrm{I}, m}, Q_{\mathrm{B}, \mathrm{AC}, \mathrm{I}, m}, P_{\mathrm{DC}, \mathrm{I}, m}$ and $V_{\mathrm{DC}, \mathrm{I}, m}$ are the ones on the inverter side, $\mu_{\mathrm{R}}$ and $X_{C, \mathrm{R}}$ are DC voltage utilization ratio and filter capacitance of the VSC station on the rectifier side, $\mu_{\mathrm{R}}$ and $X_{C, \mathrm{R}}$ are the ones on the inverter side, $\delta_{\mathrm{R}, m}$, $M_{\mathrm{R}, m}, \delta_{\mathrm{I}, m}$ and $M_{\mathrm{I}, m}$ are modulation angles and degrees of the rectifier side and inverter side of the VSC station in step $m$, $V_{\mathrm{s}, \mathrm{R}}$ and $V_{\mathrm{s}, \mathrm{I}}$, which are sent by subsystems, are voltages in subsystem boundary buses, and $Y_{\mathrm{R}}, Y_{\mathrm{I}}, \alpha_{\mathrm{I}}$ and $\alpha_{\mathrm{R}}$ satisfy equations in (A-10)-(A-11) where $Y_{\mathrm{R}}, X_{l, \mathrm{R}}, Y_{\mathrm{I}}$ and $X_{l, \mathrm{I}}$ are equivalent resistance and reactance of VSC stations on the rectifier and inverter side. Constraint (A-12) is the DC transmission limit. VSC control parameter range limits are (A-13)-(A-14).

$$
\begin{array}{r}
Y_{\mathrm{R}}=1 / \sqrt{R_{\mathrm{R}}^{2}+X_{l, \mathrm{R}}^{2}} \quad \alpha_{\mathrm{R}}=\arctan \left(R_{\mathrm{R}} / X_{l, \mathrm{R}}\right) \forall i \in V S C_{\mathrm{R}} \\
Y_{\mathrm{I}}=1 / \sqrt{R_{\mathrm{I}}^{2}+X_{l, \mathrm{I}}^{2}} \quad \alpha_{\mathrm{I}}=\arctan \left(R_{\mathrm{I}} / X_{l, \mathrm{I}}\right) \forall i \in V S C_{\mathrm{I}}( \\
\left|P_{\mathrm{DC}, \mathrm{R}}\right| \leq p_{j k, \text { max }} \\
\delta_{\mathrm{R}, \text { min }} \leq \delta_{\mathrm{R}, m} \leq \delta_{\mathrm{R}, \max } \& \delta_{\mathrm{I}, \text { min}} \leq \delta_{\mathrm{I}, m} \leq \delta_{\mathrm{I}, \max }( \\
M_{\mathrm{R}, \min } \leq M_{\mathrm{R}, m} \leq M_{\mathrm{R}, \max } \& M_{\mathrm{I}, \text { min }} \leq M_{\mathrm{I}, m} \leq M_{\mathrm{I}, \max }(
\end{array}
$$

3) Model of LCC-HVDC link: The steady-state power flow equations of the LCC-HVDC link are (A-15)-(A-24) [14], [26]. Constraints (A-25)-(A-28) are variable limits.

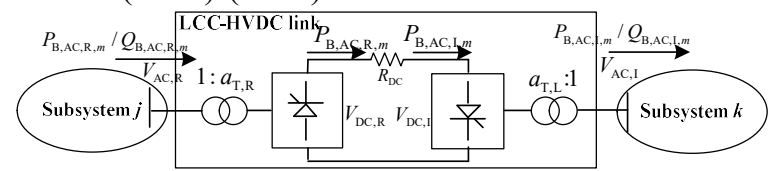

Fig. A-2 The equivalent circuit of the LCC-HVDC link

$$
\begin{array}{r}
P_{\mathrm{B}, \mathrm{AC}, \mathrm{R}, m}=V_{\mathrm{DC}, \mathrm{R}, m} I_{\mathrm{DC}, m}(\mathrm{~A}-15) \\
P_{\mathrm{B}, \mathrm{AC}, \mathrm{I}, m}=V_{\mathrm{DC}, \mathrm{I}, m} I_{\mathrm{DC}, m}(\mathrm{~A}-16) \\
Q_{\mathrm{B}, \mathrm{AC}, \mathrm{R}, m}^{2}=S_{\mathrm{R}, m}^{2}-P_{\mathrm{B}, \mathrm{AC}, \mathrm{R}, m}^{2}(\mathrm{~A}-17) \\
Q_{\mathrm{B}, \mathrm{AC}, \mathrm{I}, m}^{2}=S_{\mathrm{I}, m}^{2}-P_{\mathrm{B}, \mathrm{AC}, \mathrm{I}, m}^{2}(\mathrm{~A}-18) \\
S_{\mathrm{R}, m}=k \frac{3 \sqrt{2}}{\pi} n p_{\mathrm{R}} a_{\mathrm{T}, \mathrm{R}} V_{\mathrm{AC}, \mathrm{R}, m} I_{\mathrm{DC}, m}(\mathrm{~A}-19) \\
S_{\mathrm{I}, m}=k \frac{3 \sqrt{2}}{\pi} n p_{\mathrm{I}} a_{\mathrm{T}, \mathrm{I}} V_{\mathrm{AC}, \mathrm{I}, m} I_{\mathrm{DC}, m}(\mathrm{~A}-20)
\end{array}
$$$$
V_{\mathrm{DC}, \mathrm{R}, m}=\frac{3 \sqrt{2}}{\pi} n p_{\mathrm{R}} a_{\mathrm{T}, \mathrm{R}} V_{\mathrm{AC}, \mathrm{R}, m} \cos \alpha_{\mathrm{R}, m}-\frac{3}{\pi} X_{\mathrm{C}, \mathrm{R}} n p_{\mathrm{R}} I_{\mathrm{DC}, m}
$$$$
V_{\mathrm{DC}, \mathrm{I}, m}=\frac{3 \sqrt{2}}{\pi} n p_{\mathrm{I}} a_{\mathrm{T}, \mathrm{I}} V_{\mathrm{AC}, \mathrm{I}, m} \cos \alpha_{\mathrm{I}, m}-\frac{3}{\pi} X_{\mathrm{C}, \mathrm{I}} n p_{\mathrm{I}} I_{\mathrm{DC}, m}
$$$$
I_{\mathrm{DC}, m} R_{\mathrm{DC}}=V_{\mathrm{DC}, \mathrm{R}, m}-V_{\mathrm{DC}, \mathrm{I}, m}
$$

$$
\begin{array}{r}
\left|I_{\mathrm{DC}, m} V_{\mathrm{DC}, \mathrm{R}, m}\right| \leq p_{j k, \text { max }}(\mathrm{A}-24) \\
a_{\mathrm{T}, \mathrm{R}, \text { min}} \leq a_{\mathrm{T}, \mathrm{R}} \leq a_{\mathrm{T}, \mathrm{R}, \text { max }} \& a_{\mathrm{T}, \mathrm{I}, \text { min }} \leq a_{\mathrm{T}, \mathrm{I}} \leq a_{\mathrm{T}, \mathrm{I}, \text { max }}(\mathrm{A}-25) \\
I_{\mathrm{DC}, \text { min }} \leq I_{\mathrm{DC}, m} \leq I_{\mathrm{DC}, \text { max }}(\mathrm{A}-26) \\
V_{\mathrm{AC}, \mathrm{R}, \text { min}} \leq V_{\mathrm{AC}, \mathrm{R}, m} \leq V_{\mathrm{AC}, \mathrm{R}, \text { max }} \& V_{\mathrm{AC}, \mathrm{I}, \text { min}} \leq V_{\mathrm{AC}, \mathrm{I}, m} \leq V_{\mathrm{AC}, \mathrm{I}, \text { max }}(\mathrm{A}-27) \\
\alpha_{\mathrm{R}, \text { min }} \leq \alpha_{\mathrm{R}, m} \leq \alpha_{\mathrm{R}, \text { max }} \& \alpha_{\mathrm{I}, \text { min }} \leq \alpha_{\mathrm{I}, m} \leq \alpha_{\mathrm{I}, \text { max }}(\mathrm{A}-28)
\end{array}
$$

where $S_{\mathrm{R}, m}$ and $S_{\mathrm{I}, m}$ are respectively the apparent power on the rectifier side and inverter side in step $m, n p_{\mathrm{R}}$ and $n p_{\mathrm{I}}$ are the numbers of series connected bridges in the rectifier and inverter, respectively, $a_{\mathrm{T}, \mathrm{R}}$ and $a_{\mathrm{T}, \mathrm{I}}$ are transformer tap ratios for the rectifier side and inverter side, $\alpha_{\mathrm{R}, m}$ and $\alpha_{\mathrm{I}, m}$ are the ignition angle for rectifier operation and extinction angle for inverter operation in step $m$, and $I_{\mathrm{DC}, m}$ is the DC current in step $m$. Parameter $k$ can be determined according to [26].

4) Operation constrains between steps for HVDC links.

Because the power flow through HVDC links relies on the control of electronic devices within the LCC or VSC station, several constraints are built to match the sequential operation characteristic of HVDC links in the restoration process. Equations (A-29) and (A-30) are constraints for power adjustment of HVDC links, where binary variables $x_{C, m}^{+}$and $x_{C, m}^{-}$mean that the power flow through the HVDC link $C_{i}$ is adjusted upward or downward in step $m . M_{C}$ is the allowed number of power adjustment in restoration period $T_{M}$. To avoid repeated smallamplitude adjustments of HVDC links, (A-31) is set to ensure the power adjustment is within bounds. Therein, $r_{d c, m}^{+}$and $\dot{r_{d c, m}}$ are upward and downward adjustment bounds, respectively. Specifically, owing to the difficulty of the LCC station to reverse the power flow direction, (A-32)-(A-33) are set to avoid frequent power flow reversal, where binary variable $o_{\mathrm{B}, i, m}=1$ when the power flow is in forward direction while $o_{\mathrm{B}, i, m}=0$ when the power flow is reverse, $M_{o}$ is the constant with a large value. $N_{\mathrm{LCC}}$ and $N_{\mathrm{VSC}}$ are sets of LCC-HVDC and VSC-HVDC links.

$$
\begin{gathered}
x_{C_{i}, m}^{+}+x_{C_{i}, m}^{-} \leq 1 \quad\left(\forall C_{i} \in N_{\mathrm{LCC}} \& N_{\mathrm{VSC}}\right) \\
\sum_{m=1}^{T_{M}}\left(x_{C_{i}, m}^{+}+x_{C_{i}, m}^{-}\right) \leq M_{C}\left(\forall C_{i} \in N_{\mathrm{LCC}} \& N_{\mathrm{VSC}}\right) \\
x_{C_{i}, m}^{+} r_{d c, \text { min }}^{+}-x_{C_{i}, m}^{-} r_{i d c, \max }^{-} \leq p_{C_{i}, j k, m}-p_{C_{i}, j k, m-1} \\
\leq x_{C_{i}, m}^{+} r_{d c, \text { max }}^{+}-x_{C_{i}, m}^{-} r_{d c, \text { min }}^{-}\left(\forall C_{i} \in N_{\mathrm{LCC}} \& N_{\mathrm{VSC}}\right) \\
\left|o_{C_{i}, m}-o_{C_{i}, i, m-1}\right|+\sum_{t=m+1}^{\min \left(t_{m}, t_{m}^{+}+N_{T}\right)}\left|o_{C_{i}, t}-o_{C_{i}, t-1}\right| \leq 1\left(\forall C_{i} \in N_{\mathrm{LCC}}\right) \\
\left(o_{C_{i}, m}-1\right) M_{o} \leq I_{\mathrm{DC}, C_{i}, m} \leq o_{C_{i}, m} M_{o}\left(\forall C_{i} \in N_{\mathrm{LCC}}\right)
\end{gathered}
$$

5) Constraints for HVAC tie-line. The active power limit of tie-lines is (A-34) with the upper bound $P_{j k \text { max }}$. For HVAC tielines, the maximum phase angle difference $\theta_{j k, \max }$ is required in (A-35) to avoid excessive standing phase angle problem of neighboring subsystems reconnection.

$$
\begin{array}{r}
\left|P_{C_{i}, j k, m}\right| \leq P_{j k, \text { max }}\left(C_{i} \in N_{\mathrm{HVAC}}\right) \\
\left|\theta_{C_{i}, j, m}-\theta_{C_{i}, k, m}\right| \leq \theta_{j k, \text { max }}\left(C_{i} \in N_{\mathrm{HVAC}}\right)
\end{array}
$$

where $P_{C i, j, m}, \theta_{C i, j, m}$ and $\theta_{C i, k, m}$ are active power flow through HVAC tie-line $C_{i}$ between subsystem $j$ and $k$, phase angle on the two ends of tie-line connected to subsystem $j$ and subsys- 
tem $k . N_{\text {hvac }}$ is the set of HVAC tie-lines.

6) Model of subsystem $j$. Subsystems are with meshed network, restarted generators with minimum generation levels and un-served loads. Inequality (A-36) ensures the load will not be shed as long as it is picked up. Equation (A-37) is the multi-step generator output constraint which means generators are ramping up with ramp rate $r_{i}$ between the upper and lower limits. Equalities (A-38) and (A-39) determine the active and reactive power of each node in subsystem $j$, respectively. Constraints (A-40)-(A-46) are for the linearized AC power flow calculation. The branch flow limit (A-47)-(A-50), voltage limit (A-51) and reserve constraints (A-52)-(A-53) are security constraints. The boundary power limit is (A-54). The details of the subsystem constraints can be found in [27].

$$
\begin{aligned}
& x_{\mathrm{L}, j, i, m} \geq x_{\mathrm{L}, j, i, m-1}\left(\forall i \in N_{\mathrm{L}, j}\right) \\
& p_{\mathrm{G}, i, m} \geq \max \left\{p_{\mathrm{G}, i, m-1}+r_{i} T_{m}, p_{\mathrm{G}, i, \min }\right\} \\
& p_{\mathrm{G}, i, m} \leq \min \left\{p_{\mathrm{G}, i, m-1}+r_{i} T_{m}, p_{\mathrm{G}, i, \max }\right\} \forall i \in N_{\mathrm{G}, j} \\
& p_{n, m}=-x_{\mathrm{L}, j, i, m} p_{\mathrm{L}, j, i}\left(i \in N_{\mathrm{L}, j}\right) \|-P_{\mathrm{B}, j, i, m}\left(i \in N_{\mathrm{B}, j}\right) \\
& \| p_{\mathrm{G}, i, m}\left(i \in N_{\mathrm{G}, j}\right)+0 \\
& q_{n, m}=-x_{\mathrm{L}, j, i, m} q_{\mathrm{L}, i}\left(i \in N_{\mathrm{L}, j}\right) \| q_{\mathrm{G}, j, i, m}\left(i \in N_{\mathrm{G}, j}\right) \\
& \left\|Q_{\mathrm{B}, j, i, m}\left(i \in N_{\mathrm{B}, j}\right)\right\| 0 \\
& p_{n, m}=\sum_{\langle n, l\rangle \in N_{\text {Line }}} P_{n l, m} \forall n \in N_{\text {Subj }} \\
& q_{n, m}=\sum_{\langle n, l\rangle \in N_{\text {Line }}} Q_{n l, m} \forall n \in N_{S u b j} \\
& P_{n l, m}=g_{n l}-g_{n l} \cos ^{*} \theta_{n l, m}-b_{n l}\left(\theta_{n, m}-\theta_{l, m}\right) \\
& Q_{n l, m}=-b_{n l}-g_{n l}\left(\theta_{n, m}-\theta_{l, m}\right)+b_{n l} \cos ^{*} \theta_{n l, m}-b_{n l}\left(\delta_{n, m}-\delta_{l, m}\right)(\mathrm{A}-43) \\
& \cos ^{*} \theta_{n l, m} \geq \cos \theta_{\Delta}^{0} \\
& \cos ^{*} \theta_{n l, m} \leq-\sin \left(v d-\theta_{\Delta}^{0}\right)\left(\theta_{n, m}-\theta_{l, m}-v d+\theta_{\Delta}^{0}\right) \\
& +\cos \left(v d-\theta_{\Delta}^{0}\right) \quad v=1,2, \ldots, 2 h \\
& V_{n, m}=1+\delta_{n, m} \quad \text { (Volts p.u.) } \\
& -S_{n l, \text { max }} \leq P_{n l, m} \leq S_{n l, \text { max }} \\
& -S_{n l, \max } \leq Q_{n l, m} \leq S_{n l, \max } \\
& -\sqrt{2} S_{n l, \max } \leq P_{n l, m}+Q_{n l, m} \leq \sqrt{2} S_{n l, \text { max }} \\
& -\sqrt{2} S_{n l, \text { max }} \leq P_{n l, m}-Q_{n l, m} \leq \sqrt{2} S_{n l, \text { max }} \\
& V_{n, \min }<V_{n, m}<V_{n, \max } \quad \forall n \in N_{S u b j} \\
& \Delta f_{j, m}=\left(\Delta p_{\mathrm{TL}, j, m}+\Delta P_{\mathrm{B}, j, m}\right) / \sum_{i^{e} \in N_{\mathrm{G}, j}, i \in N_{\mathrm{G}, j}, i^{e} \neq i} \frac{s_{\mathrm{G}, j, i^{e}}}{\varepsilon_{\mathrm{G}, j, i}} \leq \max \Delta f \\
& \left\{\begin{array}{l}
\Delta p_{\mathrm{L}, j, m}=\sum_{i \in N_{\mathrm{L}, j}}\left(x_{\mathrm{L}, j, i, m} p_{\mathrm{L}, j, i, m}-x_{\mathrm{L}, j, i, m-1} p_{\mathrm{L}, j, i, m}\right) \\
\Delta P_{\mathrm{B}, j, m}=\sum_{i \in N_{\mathrm{B}, j}}\left(P_{\mathrm{B}, j, i, m}-P_{\mathrm{B}, j, i, m-1}\right)
\end{array}\right. \\
& p_{\mathrm{G}, j, i, m} \leq \sum_{i^{e} \in N_{\mathrm{G}}, l^{e} \neq i}\left(p_{\mathrm{G}, j, l^{e}, m, \text { max }}-p_{\mathrm{G}, j, i^{e}, m, \text { min }}+p_{\mathrm{G}, j, l^{e}, m}\right) \\
& -\sum_{i \in N_{\mathrm{L}, j}}\left(x_{\mathrm{L}, j, i, m} p_{\mathrm{L}, i}\right)-\sum_{i \in N_{\mathrm{B}, j}} P_{\mathrm{B}, j, i, m} \\
& -P_{\mathrm{B}, j, \text { max }} \leq P_{\mathrm{B}, j, i, m} \leq P_{\mathrm{B}, j, \text { max }}
\end{aligned}
$$

where $p_{\mathrm{G}, i, m}$ and $p_{\mathrm{G}, i, m-1}$ are the output of generator $i$ in step $m$ and $m-1, r_{i}$ is the ramping up rate of generator $i, T_{m}$ is the load pickup operation time in step $m, p_{n, m}$ and $q_{n, m}$ are the active and reactive power injection into bus $n$ in step $m, P_{n l, m}, Q_{n l, m}$ and $\mathrm{S}_{n l, m}$ are the active power flow, reactive power flow and apparent power on line $n l, g_{n l, m}, b_{n l, m}$ and $\theta_{n l, m}$ are the conductance, susceptance and phase angle difference of line $n l, V_{n, m}$, $\delta_{n, m}$ represents the voltage amplitude and voltage deviation of bus $n, \cos ^{*} \theta_{n l, m}$ is the approximate variable of $\cos \theta_{n l, m}, \Delta$ denotes the incremental change of variables, $\Delta f, \Delta p_{\mathrm{TL}, j, m}$ and $\Delta P_{\mathrm{B}, j, m}$ are the frequency deviation, load amount increase and boundary power increase in subsystem $j$ in step $m$, and $P_{\mathrm{B}, j, m}$ and $P_{\mathrm{B}, j \text { max }}$ are the boundary active power and maximum boundary active power of subsystem $j . N_{\mathrm{G}, j}, N_{\mathrm{L}, j}, N_{\mathrm{B}, j}, N_{S u b j}$ and $N_{\text {Line }}$ are sets of generator nodes, load nodes, boundary nodes, subsystem nodes and transmission lines in subsystem $j$.

7) Boundary constraints. Some boundary constraints should be satisfied when subsystems are reconnected. Eq. (A55)-(A-57) are the boundary variable consistency required between subsystem and VSC-HVDC links, subsystem and LCC-HVDC links and subsystem and HVAV tie-lines.

$$
\begin{array}{r}
P_{\mathrm{B}, \mathrm{AC}, \mathrm{C}_{i}, m}=P_{\mathrm{B}, j, i, m} \& Q_{\mathrm{B}, \mathrm{AC}, \mathrm{C}_{i}, m}=Q_{\mathrm{B}, j, i, m} \\
C_{i} \in N_{v s c}, i \in N_{\mathrm{B}, j, v s c} \\
P_{\mathrm{B}, \mathrm{AC}, \mathrm{C}_{i}, m}=P_{\mathrm{B}, j, i, m} \& Q_{\mathrm{B}, A C, \mathrm{C}_{i}, m}=Q_{\mathrm{B}, j, i, m} \& V_{\mathrm{B}, \mathrm{AC}, \mathrm{C}_{i}, m}=V_{\mathrm{B}, j, i, m} \\
C_{i} \in N_{\mathrm{LCC}}, i \in N_{\mathrm{B}, j, l, c c} \\
P_{\mathrm{C}_{i}, j k, m}=P_{\mathrm{B}, j, i, m}=P_{\mathrm{B}, k, i, m} \& \theta_{C_{i}, j, m}=\theta_{\mathrm{B}, j, i, m} \\
C_{i} \in N_{h v a c}, i \in N_{\mathrm{B}, j, h v a c}
\end{array}
$$

Note that models of HVDC links, HVAC tie-lines and subsystems are built for the multi-step process where step $m \in$ $\left\{1, \ldots, T_{M}\right\}$.

\section{Appendix B}

The convergence analysis of HOC_ATC is as follows.

Set the original problem (1) as (B-1). Based on (B-1), the sub-problems in blocks A and B are (B-2) and (B-3), respectively. $\boldsymbol{Y}_{\mathrm{a}}$ and $\boldsymbol{Y}_{\mathrm{b}}$ are linking variables between two blocks, and $\boldsymbol{Y}_{\mathrm{o}}$ represents other variables.

$$
\begin{array}{ll}
\min & f\left(\boldsymbol{Y}_{\mathrm{a}}, \boldsymbol{Y}_{\mathrm{b}}, \boldsymbol{Y}_{\mathrm{o}}\right) \\
\text { s.t. } & \boldsymbol{Y}_{\mathrm{a}} \in \Theta_{1}, \boldsymbol{Y}_{\mathrm{b}} \in \Theta_{2}, \boldsymbol{Y}_{\mathrm{o}} \in \Theta_{3} \\
\min & f\left(\boldsymbol{Y}_{\mathrm{a}}, \boldsymbol{Y}_{\mathrm{b}}, \boldsymbol{Y}_{\mathrm{o}}\right) \\
\text { s.t. } & \boldsymbol{Y}_{\mathrm{a}} \in \Theta_{1}, \boldsymbol{Y}_{\mathrm{b}}=\boldsymbol{Y}_{\mathrm{b}}^{*}, \boldsymbol{Y}_{\mathrm{o}} \in \Theta_{3} \\
\min & f\left(\boldsymbol{Y}_{\mathrm{a}}, \boldsymbol{Y}_{\mathrm{b}}, \boldsymbol{Y}_{\mathrm{o}}\right) \\
\text { s.t. } & \boldsymbol{Y}_{\mathrm{a}}=\boldsymbol{Y}_{\mathrm{a}}^{*}, \boldsymbol{Y}_{\mathrm{b}} \in \Theta_{2}, \boldsymbol{Y}_{\mathrm{o}} \in \Theta_{3}
\end{array}
$$

The HOC_ATC starts with block B based on any feasible $\boldsymbol{Y}_{\mathrm{a}}^{*}$ $=\boldsymbol{Y}_{\mathrm{a}}^{\mathrm{\perp} \mathrm{B}}$. In block B, (B-3) is further decomposed into the parallel subsystem model (B-4) and tie-line model (B-5).

$$
\begin{array}{ll}
\min & f_{\mathrm{b}, \text { Sub }}\left(\boldsymbol{Y}_{\mathrm{a}}, \boldsymbol{Y}_{\mathrm{b}}, \boldsymbol{Y}_{\mathrm{o}}\right) \\
\text { s.t. } & \boldsymbol{Y}_{\mathrm{a}}=\boldsymbol{Y}_{\mathrm{a}}^{*}, \boldsymbol{Y}_{\mathrm{b}} \in \Theta_{2}, \boldsymbol{Y}_{\mathrm{o}} \in \Theta_{3} \\
\min & f_{\mathrm{b}, \text { Tie }}\left(\boldsymbol{Y}_{\mathrm{a}}, \boldsymbol{Y}_{\mathrm{b}}, \boldsymbol{Y}_{\mathrm{o}}\right) \\
\text { s.t. } & \boldsymbol{Y}_{\mathrm{a}}=\boldsymbol{Y}_{\mathrm{a}}^{*}, \boldsymbol{Y}_{\mathrm{b}} \in \Theta_{2}, \boldsymbol{Y}_{\mathrm{o}} \in \Theta_{3}
\end{array}
$$

In block B, the ATC algorithm is performed based on these parallel models to obtain the optimization result in (B-6). Note that subsystem models (B-4) is a QP problem since binary 
variables have been fixed in $\boldsymbol{Y}_{\mathrm{a}}$. Thus, if the tie-line model (B5 ) is convex, the result of applying the ATC method can be strictly proved to be equal to the optimal result of (B-3) [24]. In this paper, non-linear constraints of HVDC tie-line models are used. Despite this, the HVDC models are in small-scale forms with several non-linear constraints so that the ATC algorithm within block B can still provide the result with a small optimization gap (within $0.01 \%$ as shown in the case study of [16]). Accordingly, the in-equality in (B-6) can be satisfied.

$$
\begin{aligned}
& f\left(\boldsymbol{Y}_{\mathrm{a}}^{L_{\mathrm{AB}}}, \boldsymbol{Y}_{\mathrm{b}}^{L_{\mathrm{AB}}}, \boldsymbol{Y}_{\mathrm{o}}^{L_{\mathrm{AB}}}\right) \\
& \leq\left\{f\left(\boldsymbol{Y}_{\mathrm{a}}^{L_{\mathrm{AB}}}, \boldsymbol{Y}_{\mathrm{b}}, \boldsymbol{Y}_{\mathrm{o}}\right) \text { s.t. } \boldsymbol{Y}_{\mathrm{b}} \in \Theta_{2}, \boldsymbol{Y}_{\mathrm{o}} \in \Theta_{3}\right\}
\end{aligned}
$$

Then, the obtained $\boldsymbol{Y}_{\mathrm{b}}^{\mathrm{LAB}_{\mathrm{B}}}$ becomes $\boldsymbol{Y}_{\mathrm{b}}^{*}$ for block A. In block A, (B-2) is decomposed into the parallel subsystem model (B-7) and tie-line equations (B-8). The optimal objective value of (B-2) can be obtained by optimizing (B-7).

$$
\begin{array}{cl}
\min & f_{\mathrm{a}, \text { Sub }}\left(\boldsymbol{Y}_{\mathrm{a}}, \boldsymbol{Y}_{\mathrm{b}}, \boldsymbol{Y}_{\mathrm{o}}\right) \\
\text { s.t. } & \boldsymbol{Y}_{\mathrm{a}} \in \Theta_{1}, \boldsymbol{Y}_{\mathrm{b}}=\boldsymbol{Y}_{\mathrm{b}}^{*}, \boldsymbol{Y}_{\mathrm{o}} \in \Theta_{3} \\
\text { E.q. } \boldsymbol{g}_{\mathrm{a}, \mathrm{Tie}}\left(\boldsymbol{Y}_{\mathrm{b}}, \boldsymbol{Y}_{\mathrm{o}}\right) \leq 0 \\
\qquad \boldsymbol{Y}_{\mathrm{b}}=\boldsymbol{Y}_{\mathrm{b}}^{*}, \boldsymbol{Y}_{\mathrm{o}} \in \Theta_{3}
\end{array}
$$

With fixed boundary variables in $\boldsymbol{Y}_{\mathrm{b}}^{*}\left(\boldsymbol{Y}_{\mathrm{b}}^{L_{\mathrm{AB}}}=\boldsymbol{Y}_{\mathrm{b}}^{*}\right)$, the optimization of (B-7) is MIQP and tie-line models do not need optimization. Solving (B-7) and (B-8), variables in $\boldsymbol{Y}_{\mathrm{a}}$ and $\boldsymbol{Y}_{\mathrm{o}}$ are with fixed values in $\boldsymbol{Y}_{\mathrm{a}}^{L_{\mathrm{AB}}+1}$ and $\boldsymbol{Y}_{\mathrm{o}}^{L_{\mathrm{A}}+1}$. The MIQP model is solved by Gurobi which can guarantee the optimality using the Branch-and-Cut algorithm. Therefore, the calculation in block A can get the result satisfying (B-9) where the left hand side is the optimized objective value and the right hand side is the possible objective function values in a feasible region.

$$
\begin{aligned}
& f\left(\boldsymbol{Y}_{\mathrm{a}}^{L_{\mathrm{AB}}+1}, \boldsymbol{Y}_{\mathrm{b}}^{L_{\mathrm{AB}}}, \boldsymbol{Y}_{\mathrm{o}}^{L_{\mathrm{AB}}+1}\right) \\
\leq & \left\{f\left(\boldsymbol{Y}_{\mathrm{a}}, \boldsymbol{Y}_{\mathrm{b}}^{L_{\mathrm{AB}}}, \boldsymbol{Y}_{\mathrm{o}}\right) \text { s.t. } \boldsymbol{Y}_{\mathrm{a}} \in \Theta_{1}, \boldsymbol{Y}_{\mathrm{o}} \in \Theta_{3}\right\}
\end{aligned}
$$

Get $\boldsymbol{Y}_{\mathrm{a}}^{*}=\boldsymbol{Y}_{\mathrm{a}}^{L_{\mathrm{AB}}+1}$, the second round of block B has (B-10).

$$
\begin{aligned}
& f\left(\boldsymbol{Y}_{\mathrm{a}}^{L_{\mathrm{AB}}+1}, \boldsymbol{Y}_{\mathrm{b}}^{L_{\mathrm{AB}}+1}, \boldsymbol{Y}_{\mathrm{o}}^{L_{\mathrm{AB}}+1}\right) \\
\leq & \left\{f\left(\boldsymbol{Y}_{\mathrm{a}}^{L_{\mathrm{AB}}+1}, \boldsymbol{Y}_{\mathrm{b}}, \boldsymbol{Y}_{\mathrm{o}}\right) \text { s.t. } \boldsymbol{Y}_{\mathrm{b}} \in \Theta_{2}, \boldsymbol{Y}_{\mathrm{o}} \in \Theta_{3}\right\}
\end{aligned}
$$

According to (B-10) and (B-9), (B-11) is satisfied. It means the proposed HOC_ATC algorithm has the convergence attribute by providing the iteratively decreasing result for the minimization problem. Since the minimizing problem at least has a lower bound, the iteration converges if the objective value keeps on decreasing.

$$
f\left(\boldsymbol{Y}_{\mathrm{a}}^{L_{\mathrm{AB}}+1}, \boldsymbol{Y}_{\mathrm{b}}^{L_{\mathrm{AB}}+1}, \boldsymbol{Y}_{\mathrm{o}}^{L_{\mathrm{AB}}+1}\right) \leq f\left(\boldsymbol{Y}_{\mathrm{a}}^{L_{\mathrm{AB}}}, \boldsymbol{Y}_{\mathrm{b}}^{L_{\mathrm{AB}}}, \boldsymbol{Y}_{\mathrm{o}}^{L_{\mathrm{AB}}}\right)(\mathrm{B}-11)
$$

\section{REFERENCES}

[1] F. Qiu and P. Li, "An Integrated Approach for Power System Restoration Planning," Proc. IEEE, vol. 105, no. 7, pp. 1234-1252, 2017.

[2] N.A.Fountas, N.D. Hatziargyriou, C. Orfanogiannis and A. Tasoulis, "Interactive long-term simulation for power system restoration planning," IEEE Trans. Power Syst., vol, 12 no. 1, pp. 61 -68, 1997.

[3] L. Sun, C. Peng, J. Hu and Y. Hou, "Application of Type 3 Wind Turbines for System Restoration," IEEE Trans. Power Syst., vol. 33, no. 3, pp. 3040-3051, 2018.

[4] L. H. Fink, K. L. Liou, and C. C. Liu, "From generic restoration actions to specific restoration strategies," IEEE Trans. Power Syst., vol. 10, no. 2, pp. 745-751, May 1995.
[5] S. Abbasi, M. Barati and G. J. Lim, "A parallel sectionalized restoration scheme for resilient smart grid systems," IEEE Trans. Smart Grid, vol. PP, no. 99, pp. 1-1, 2018.

[6] C. Wang, V. Vittal, and K. Sun, "OBDD-based sectionalizing strategies for parallel power system restoration,” IEEE Trans. Power Syst., vol. 26, no.3, pp.1426-1433, 2011.

[7] Y. Liu, "Analysis on and Inspiration of the "9.13" Islanding and Outage of Brazilian Remote Northwest Power Grid", Proceedings of the CSEE, vol. 38, no. 11, pp. 3204-3213, 2018.

[8] M. Bahrman and P. E. Bjorklund, "The new black-start- system restoration with help from volt-age-sourced converter," IEEE Power Energy Mag., vol. 12, no.1, pp. 45-53, 2014.

[9] S. Barsali, R. Salvati and R. Zaottini. "Use of HVDC links for power system restoration," Electric Power Syst. Research, vol. 79, no.6, pp. 973-983, 2009.

[10] L. Chen, C. Wang and Q. Zhou, "A multi-objective optimization method of black-start restoration utilizing HVDC links," IEEE PES Generl Meeting, pp. 1-5, 2016.

[11] C. Liu, B. Zhang, Y. Hou, F. F. Wu and Y. Liu, "An improved approach for AC/DC power flow calculation with multi-infeed DC Systems," IEEE Trans. Power Syst. vol. 26, no. 2, pp. 862-869, 2011.

[12] T. Smed, G. Andersson, G. B. Sheble and L. L. Grigsby, "A new approach to AC/DC power flow," IEEE Trans. Power Syst. vol. 6, no.3, pp. 1238-1244, 1991.

[13] A. Lotfjou, Y. Fu and M. Shahidehpour, "Hybrid AC/DC transmission expansion planning," IEEE Trans. Power Del. vol. 27, no.3, pp. 1620$1628,2012$.

[14] M. E. M. Djesus, D. S. Martin S. Arnalters and E. D. Castronuovo, "Optimal operation of offshore wind farms with line-commutated HVDC link connection," IEEE Trans. Energy Convers., vol. 25, no. 2, pp. 504-513, 2010.

[15] K. Murari and N. P. Padhy, "A network-topology-based approach for the load-flow solution of AC-DC distribution system with distributed Generations," IEEE Trans. Ind. Informat., vol. 15, no. 3, pp. 1508-1520, 2019.

[16] C. Qi, K. Wang, Y. Fu, G. Li, B. Han, R. Huang and T. Pu, "A decentralized optimal operation of AC/DC hybrid distribution grids," IEEE Trans. Smart Grid, vol. 9, no. 6, pp. 6095-6105, 2018.

[17] J. Zhao, H. Wang, Y. Liu, Q. Wu, Z. Wang and Y. Liu, "Coordinated restoration of transmission and distribution system using decentralized scheme," IEEE Trans. Power Syst., to be published.

[18] S. Huang, Q. Wu, Y. Guo, X. Chen, B. Zhou and C. Li, "Distributed voltage control based ADMM for large-scale wind farm cluster connected to VSC-HVDC," IEEE Trans. Sustain. Energy, vol. 11, no. 2, pp. 584-594, 2019.

[19] S. Huang, Q. Wu, J. Zhao, W. Liao, "Distributed optimal voltage control for VSC-HVDC connected large-scale wind farm cluster based on analytical target cascading method," IEEE Trans. Sustain. Energy, 2019, in press.

[20] M. Zhou, J. Zhai, G. Li and J. Ren, "Distributed dispatch approach for bulk AC/DC hybrid Systems with high wind power penetration," IEEE Trans. Power Syst., vol. 33, no.3, pp. 3325-3336, 2018.

[21] Y. Zhao, Z. Lin, Y. Ding, Y. Liu, L. Sun and Y. Yan, "A model predictive control based generator start-up optimization strategy for restoration with microgrids as black-start resources," IEEE Trans. Power Syst., vol. 33, no.6, pp. 7189-7203, 2018.

[22] A. Gholami and F. Aminifar, "A hierarchical response-based approach to the load restoration problem," IEEE Trans. Smart Grid, vol. 8, no. 4, pp. 1700-1709, 2017.

[23] G. Monique and S. Kim, "Lagrangean decomposition: A model yielding stronger Lagrangean bounds," Math. Program., vol. 39, no. 2, pp. 215228, 1987.

[24] S. Tosserams, L. F. P. Etman, P. Y. Papalambros, and J. E. Rooda, “An augmented Lagrangian relaxation for analytical target cascading using the alternating directions method of multipliers," Struct. Multdisc. Optim., vol. 31, no. 3, pp. 176-189, 2006.

[25] N. Michelena and P. Papalambros, H. A. Park and D. Kulkarni, "Hierarchical overlapping coordination for large-scale optimization by decomposition" AIAA Journal, vol. 37, no. 7, pp. 890-896, 1991.

[26] H. Ambriz-Perez, E. Acha and C.R. Fuerte-Esquivel, "High voltage direct current modelling in optimal power flows," Int. J. Electr. Power Energy Syst., vol. 30, no. 3, pp. 157-168, 2008.

[27] J. Zhao, H. Wang, Y. Liu, R. Azizipanah-Abarghooee and V. Terzija, "Utility-oriented online load restoration considering wind power penetration," IEEE Trans. Sustain. Energy, vol. 10, no. 2, pp. 706-717, 2019. 
[28] H. M. A. Ahmed, A. B. Eltantawy and M. M. A. Salama, "A planning approach for the network configuration of AC-DC hybrid distribution systems," IEEE Trans. Smart Grid, vol. 9, no. 3, pp. 2203-2213, 2018.

[29] W. Feng, L. A. Tuan, L. B. Tjernberg, A. Mannikoff and A. Bergman, "A new approach for benefit evaluation of multiterminal VSC-HVDC using a proposed mixed AC/DC optimal power flow," IEEE Trans. on Power Deliv., vol. 29, no. 1, pp. 432-443, 2014.

[30] A. Rabiee, A. Soroudi and A. Keane, "Risk-averse preventive voltage control of AC/DC power systems including wind power generation," IEEE Trans. Sustain. Energy, vol. 6, no. 4, pp. 1494-1505, 2015.

[31] A. Kargarian, Y. Fu and Z. Li, "Distributed security-constrained unit commitment for large-scale power systems," IEEE Trans. Power Syst., vol. 30, no. 4, pp. 1925-1936, 2015.

[32] T. Jain, D. Ghosh, D. K. Mohanta. Augmentation of situational awareness by fault passage indicators in distribution network incorporating network reconfiguration. Protectionand Control of Modern Power Systems, 4(4): 323-336, 2019.

[33] C. Coffrin and P. V. Hentenryck, "A linear-programming approximation of AC power flows," INFORMS J. Comput., vol. 26, no. 4, pp. 718-734, May 2014.

[34] C. Coffrin and P. V. Hentenryck, "Transmission system restoration with co-optimization of repairs, load pickups, and generation dispatch," Int. J. Elect. Power Energy Syst., vol. 72, pp. 144-154, Nov. 2015.

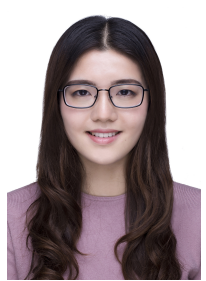

Jin Zhao (S'17) received the B.Sc. degree in electrical engineering in 2015 from Shandong University, Jinan, China, where she is working toward the Ph.D. degree in electrical engineering. She was a research assistant at The University of Hong Kong, China, from Sep. 2018 to Dec. 2018. From 2018 to 2019 , she was a visiting student at the Department of Electrical Engineering, Technical University of Denmark. Her research interests include power system resilience, restoration, renewable energy integration, and power system optimization.

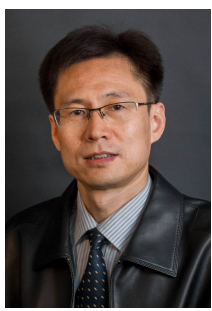

Hongtao Wang (M'04-SM'15) received the B.Sc. and M.Sc. degrees in electrical engineering from Shandong Polytechnic University, Jinan, China, in 1995 and 1998, respectively, and the $\mathrm{Ph} . \mathrm{D}$. degree in electrical engineering from Shandong University, Jinan, in 2005.

He was a Postdoctoral Researcher with the Department of Computer Science, Shandong University. From 2005 to 2010, he was an Associate Professor with the School of Electrical Engineering, Shandong University, where he has been a Professor since 2010. His research interests include power system analysis and control and smart grids.

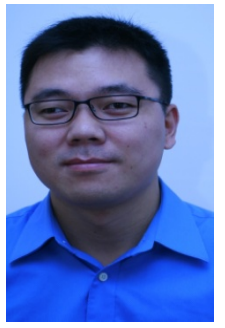

Qiuwei Wu (M'08-SM'15) obtained the PhD degree in Power System Engineering from Nanyang Technological University, Singapore, in 2009.

He was a senior R\&D engineer with VESTAS Technology R\&D Singapore Pte Ltd from Mar. 2008 to Oct. 2009. He has been working at Department of Electrical Engineering, Technical University of Denmark (DTU) since Nov. 2009 (PostDoc Nov. 2009-Oct. 2010, Assistant Professor Nov. 2010-Aug. 2013, Associate Professor since Sept. 2013). He was a visiting scholar at Department of Industrial Engineering \& Operations Research (IEOR), University of California, Berkeley, from Feb. 2012 to May 2012 funded by the Danish Agency for Science, Technology and Innovation (DASTI), Denmark. He was a visiting professor named by Y. Xue, an Academician of Chinese Academy of Engineering, at Shandong University, China, from Nov. 2015 to Oct. 2017. He was a visiting scholar at the Harvard China Project, School of Engineering and Applied Sciences, Harvard University from Nov. 2017 - Oct. 2018 funded by the Otto Mønsted Fond. His research interests are operation and control of power systems with high penetration of renewables, including wind power modelling and control, active distribution networks, and operation of integrated energy systems. He is an Editor of IEEE Transactions on Smart Grid and IEEE Power Engineering Letters. He is also an Associate Editor of International Journal of Electrical Power and Energy Systems, and Journal of Modern Power Systems and Clean Energy. He is a subject editor for IET Generation, Transmission \& Distribution, and IET Renewable Power Generation.

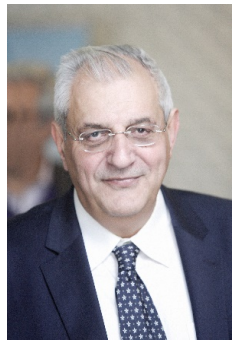

Nikos D. Hatziargyriou is full professor in Power Systems at the Electrical and Computer Engineering School of the National Technical University of Athens. From 2015 to 2019 he was Chair and until 2018, CEO of the Hellenic Distribution Network Operator (HEDNO). From 2007 until 2012 he was executive Vice-Chair and Deputy CEO of the Public Power Corporation (PPC), responsible for the Transmission and Distribution Divisions. He is Fellow Member of IEEE, past Chair of the Power System Dynamic Performance Committee (PSDPC) and currently Editor in Chief of the IEEE Trans on Power Systems. He is honorary member of CIGRE and past Chair of CIGRE SC C6 "Distribution Systems and Distributed Generation". He was chair and currently vice-chair of the EU Technology and Innovation Platform on Smart Networks for Energy Transition (ETIP-SNET). He has participated in more than $60 \mathrm{RD} \& \mathrm{D}$ projects funded by the EU Commission, electric utilities and manufacturers. He is author of the book "Microgrids: Architectures and Control" and of more than 250 journal publications and 500 conference proceedings papers. He is included in the 2016, 2017 and 2019 Thomson Reuters lists of the top $1 \%$ most cited researchers.

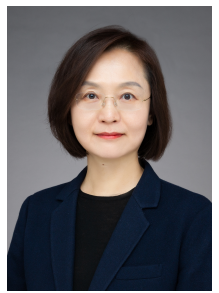

Wen Zhang received the B.S., M.S., and Ph.D. degrees from Shandong University, Jinan, China, in 1989, 1992, and 2006, respectively, all in Electrical Engineering. She is currently a professor with the Key Laboratory of Power System Intelligent Dispatch and Control of Ministry of Education, Shandong University. Her current research interests include power system analysis and control, demand-side response, and application of artificial intelligence to power systems. 